Cytogenetic and

GenomeResearch

The abstracts are only available online, free of charge, under www.karger.com/doi/10.1159/000306449

\title{
41th Biennial American Cytogenetics Conference
}

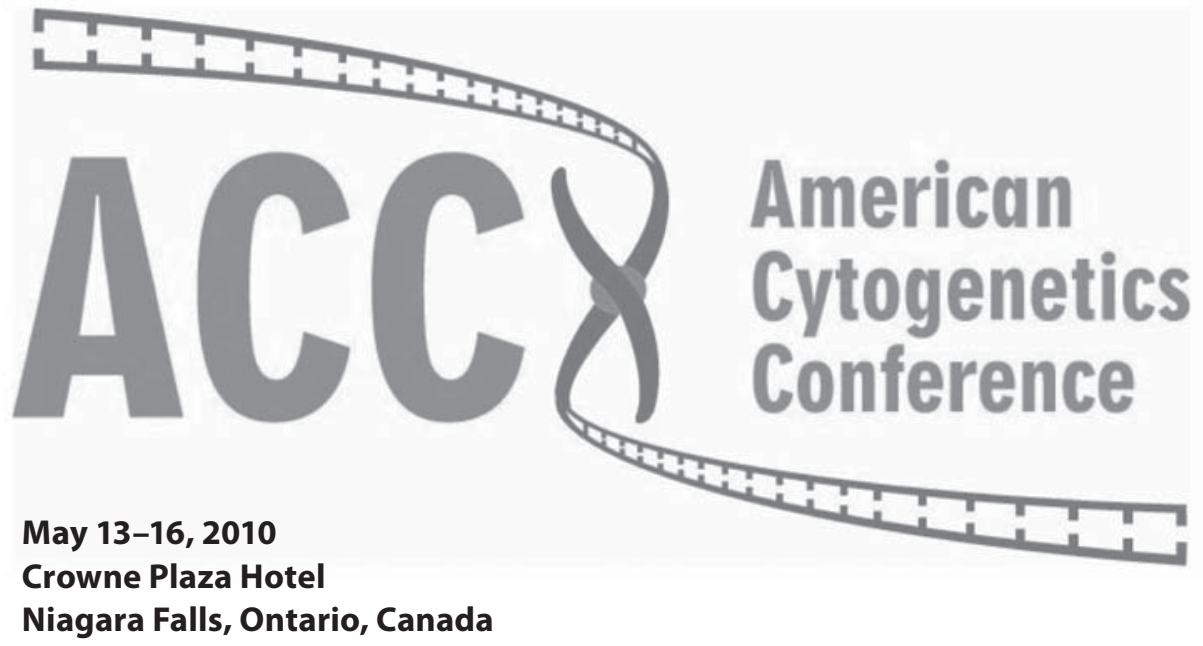




\section{1}

\section{Recurrent Chromosomal Translocations Mediated by Genomic Interchromosomal NAHR}

\author{
S.-H. Kang ${ }^{\text {a }}$, Z. Ou ${ }^{\text {a }}$, P. Stankiewicz ${ }^{\text {a }, ~ A . M . ~ B r e m a n ~}{ }^{\text {a }}$, \\ J. Wiszniewska a ,M.L. Cooper ${ }^{\text {a }}$, L. Shao ${ }^{\text {a }}$, S.T. South ${ }^{\text {b }}$, K. Coleman ${ }^{c}$, \\ P.M. Fernhoff ${ }^{c}$, M.J. Deray ${ }^{\mathrm{d}}$, S. Rosengren ${ }^{\mathrm{e}}$, E.R.Roeder $\mathrm{f}$, \\ V.B.Enciso ${ }^{f}$, A. Patel a , A. Craig Chinault a , J.R. Lupski ${ }^{\text {a, g, h, }}$ \\ S.W. Cheung ${ }^{\text {a }}$ \\ aDepartment of Molecular \& Human Genetics, Baylor College \\ of Medicine, Houston, Tex., b Departments of Pediatrics and \\ Pathology, University of Utah, Salt Lake City, Utah, 'Children's \\ Healthcare of Atlanta, Atlanta, Ga., dDepartment of Neurology, \\ Miami Children's Hospital, Miami, Fla., eHartford Hospital, \\ Hartford, Conn., fDepartment of Pediatrics, UTHSCSA, San \\ Antonio, 9Department of Pediatrics, Baylor College of Medicine, \\ and 'Texas Children's Hospital, Houston, Tex., USA \\ Z.O. and P.S. contributed equally to this work.
}

To date, the molecular mechanisms for only 2 recurrent constitutional non-Robertsonian translocations have been described: $\mathrm{t}(11 ; 22)(\mathrm{q} 23 ; \mathrm{q} 11)$ and $\mathrm{t}(4 ; 8)(\mathrm{p} 16 ; \mathrm{p} 23)$. These are mediated by either AT-rich cruciform structures or the olfactory receptor-gene cluster low-copy repeats (LCRs), respectively. We describe 3 unrelated families with an unbalanced translocation $\operatorname{der}(4) \mathrm{t}(4 ; 11)$ (p16.2;p15.4), in which the breakpoints cluster. DNA sequence analysis of the breakpoint regions revealed the presence of large 200-300 kb LCRs on 4p16.2 and 11p15.4 of 94\% interchromosomal sequence identity; the translocation breakpoints for both the short arms of chromosomes 4 and 11 map within the homologous subunits. We also identified a pericentric inversion with the $11 \mathrm{p}$ breakpoint mapping within the same LCR in 11p15.4. To investigate the potential involvement of interchromosomal LCRs in nonallelic homologous recombination (NAHR)-mediated recurrent chromosomal translocations, we performed computational genome wide analysis. We identified 470 interchromosomal LCRs, greater than $30 \mathrm{~kb}$ in size and sharing $>94 \%$ sequence identity that can potentially mediate chromosomal translocations. We constructed a global genomic view of these interchromosomal LCRs, enabling predictions of hotspots that may mediate recurrent chromosomal translocations via NAHR. Our experimental data show that interchromosomal LCRs in 11p15.4 represent a novel unstable genomic region and mediate the recurrent constitutional translocation $\mathrm{t}(4 ; 11)(\mathrm{p} 16.2 ; \mathrm{p} 15.4)$ via NAHR. Computational genome analyses reveal that hundreds of other interchromosomal LCRs can potentially mediate the formation of reciprocal translocations and other chromosomal aberrations.
2

\section{Complex Rearrangements Require Complex Analysis by Array, FISH and Karyotype}

X. Li, L.K. Conlin, S. Mulchandani, B. Thiel, M. Deardorff, E. Zackai, M. Falk, H. Hakonsarson, N. Spinner

Cytogenomics Laboratory, The Children's Hospital of

Philadelphia, 1012 ARC, Philadelphia, Pa., USA

Background: Microarray technology has dramatically increased the resolution for detecting clinically significant genomic deletions and duplications, however, it has limitations in detecting balanced translocations or completely defining complex chromosome rearrangements (CCRs). We present 4 cases in which genome-wide SNP array analysis was not sufficient to correctly diagnose a chromosomal rearrangement. In these 4 cases, SNP array analysis did demonstrate an imbalance, but further cytogenetic study revealed a more complex picture.

Case report: Case 1 is a 6 -year-old female who presented with significant developmental delay. SNP array using the Illumina Quad610 BeadChip detected a 2.35-Mb deletion on chromosome $14 \mathrm{q} 11.2 \mathrm{q} 12$ containing 70 genes. Chromosome analysis (CA) revealed an unbalanced translocation involving 3 chromosomes.

Case 2 is a 4 -year-old female with multiple congenital anomalies. Array detected a 49.2-Mb duplication on 3q24q29 containing 50 genes and a $2.83-\mathrm{Mb}$ deletion on $4 \mathrm{q} 35.2$ containing 6 genes. CA and FISH further revealed a complex 3-way unbalanced translocation between chromosomes 3, 7 and 4 .

Case 3 is a 23 -year-old male with gaze palsy, ataxia, scoliosis and myopia. Array detected a 228 -kb deletion on 20 q13.33 including 4 genes. CA and FISH demonstrated an unbalanced translocation between chromosome 17 and 20 .

Case 4 is a 19-year-old male with anomalies of skull and face bones. Array detected a 2.57-Mb deletion on 7p21.1 containing 11 genes. CA further revealed a 2 -way translocation between chromosome 2 and 7.

Conclusion: Genome-wide array analysis is highly sensitive, allowing identification of deletions and duplications much smaller than can be seen by cytogenetics alone. However, as no positional information is obtained from array studies, cytogenetics is often required to correctly describe genomic rearrangements. We recommend follow up of array findings by cytogenetic analysis as demonstrated in these 4 cases. 


\section{3}

\section{All Four Common Segregants of a Familial Balanced Translocation in Liveborn Quadruplets}

S.T. South ${ }^{\mathrm{a}-\mathrm{d}}$, L. Rowe ${ }^{\mathrm{a}, \mathrm{b}}$, D. LaGrave ${ }^{\mathrm{b}}$, R. Vanzo $^{\mathrm{c}}$, J. Carey ${ }^{\mathrm{c}}$

${ }^{a}$ Institute for Clinical and Experimental Pathology, ${ }^{\mathrm{b}} \mathrm{Associated}$ Regional and University Pathologists (ARUP) Laboratories, Departments of ${ }^{\mathrm{C} P e d i a t r i c s}$ and ${ }^{\mathrm{d} P a t h o l o g y}$, University of Utah, Salt Lake City, Utah, USA

For most balanced translocations between chromosomes A and $\mathrm{B}$, the 4 most likely segregations will lead to conceptions that are either normal or balanced due to alternate segregation, or with a partial monosomy for chromosome A with partial trisomy for chromosome B or partial monosomy for chromosome B with partial trisomy for chromosome A due to adjacent-1 segregation. Although all 4 segregations in theory are equally probable, the genetic imbalance associated with the adjacent- 1 segregation can result in lethality during development. Therefore, often not all 4 possible combinations are observed in the liveborn offspring.

We present a family with a $t(1 ; 16)(p 36.32 ; p 13.3)$ in which all 4 common segregations were observed in the liveborn quadruplets of a single pregnancy. The balanced and unbalanced versions of this translocation were cytogenetically cryptic and were unrecognized prior to the microarray analysis of one of the clinically af fected quadruplets. The father of the quadruplets was subsequently recognized as a balanced translocation carrier, as was an older sibling of the quadruplets.

In addition to the unique finding of all 4 segregants in a single pregnancy, all carriers of the balanced rearrangement and the 2 unbalanced carriers show various degrees of hearing loss, suggestive of a gene disruption or misregulation associated with the translocation. Microarray analysis of one of the unbalanced quadruplets shows the translocation either disrupts or is directly $5^{\prime}$ to the AJAP1 gene at 1 p36.32 which encodes an adherens junction associated protein involved in cell adhesion and cell migration. This finding may suggest a role for this gene in hearing. However, as the degree of hearing loss is quite variable among the different affected family members, the hearing loss may be multifactorial or due to another factor segregating in the family.

\section{4}

\section{Comparative Cytogenetics}

O. Ryder

San Diego Zoo's Institute for Conservation Research, San Diego, Calif., USA

Cytogenetic analysis remains a crucial aspect of medical diagnosis and the process of defining species and their evolution. As genomic studies diffuse into ever-widening arenas of biological inquiry, opportunities to integrate and extend cytogenetic analysis to understand important aspects of species biology, including applications to conservation, are certainly anticipated. In reviewing the early development of the field of conservation genomics, numerous references can be made to how advances in comparative cytogenetics will continue to help contribute to conservation efforts for a wide diversity of species.

\section{5 \\ Prenatal Array CGH Testing: Managing 'Abnormal' Results}

D.I. Quigley ${ }^{\text {a }}$, A.C. Holt ${ }^{\text {b }}$, C.L. Beattie ${ }^{\text {b }}$, P.B. Jacky ${ }^{\text {a }}$

Departments of a Pathology and ${ }^{\mathrm{b}}$ Genetics, Kaiser Permanente NW, Portland, Oreg., USA

Prenatal array CGH testing is increasingly being requested. Potential caveats to such testing include appropriate timing of testing during pregnancy, interpretation of findings and accurate counseling regarding anticipated outcomes. We present 2 cases in which prenatal array testing was employed and the impact of findings on the family and the pregnancy. The first case was the 4 th pregnancy for a 37-year-old woman with a history of a monozygotic twin pregnancy that was discontinued because of discrepant fetal genitalia and $46, \mathrm{XX},+S R Y$ by amnio. Routine chromosome analysis of the current pregnancy showed a normal male karyotype. Array CGH testing was requested due to maternal anxiety. Array results showed a duplication of the $\mathrm{Y}$ chromosome including the SRY locus. The duplication was shown to be a paternally inherited tandem duplication. The second case was an otherwise normal pregnancy for a G3P1 28-year-old woman. At 20 weeks gestation fetal tetralogy of Fallot, choroids plexus cysts and 2 -vessel cord were noted by ultrasound. Routine chromosome analysis of amniocytes revealed a supernumerary marker chromosome. The marker was determined to be de novo and array CGH studies were pursued to characterize the additional material. Array CGH results showed a large deletion and duplication unrelated to the marker. The pregnancy was discontinued. Follow up studies revealed that the deletion and duplication were inherited.

\section{6 \\ Detection of Homozygosity Using SNP-Based Microarrays: Case Reports and Lessons Learned \\ H.M. Kearney, C. Wilson-Brackett, C. Shanahan, S.F. Roberts, K. Crandall, J.B. Kearney, W.P. Allen, E. Boyd \\ Fullerton Genetics Center, Mission Hospitals, Asheville, N.C., USA}

SNP-based microarrays allow for the detection of copy number imbalances as well as regions of homo- and heterozygosity throughout the genome. Our center has used the Affymetrix Genome-wide Human SNP 6.0 microarray for the past 19 months in clinical service. More than $2 \%$ of our cases reveal either significant consanguinity or single chromosomes with large homozygous stretches, thus suspicious for uniparental disomy. Our laboratory reports homozygosity when it exceeds $2 \%$ of the total genome or when a single block is larger than $10 \mathrm{Mb}$. This talk will describe our experience with these cases, both illustrating the clinical utility of these findings, as well as the significant legal/ ethical and counseling issues that we have faced. 


\section{7 \\ Delineating Chromosomal Regions with Complex Architectures Using Single- and Low-Copy FISH Algorithms}

W.A. Khan a , J.H.M. Knoll ${ }^{\text {b }}$, P.K. Rogan a

Departments of aBiochemistry and bathology, University of Western Ontario, London, Ont., Canada

Segmental duplications (SDs) enriched in mammalian genomes exhibit predisposition for aberrant chromosomal structural changes that account for the majority of the burden in human congenital disease. The importance of being able to detect relevant genomic architecture generating recurrent and non-recurrent chromosomal rearrangements is significant, because a diverse range of phenotypes can manifest due to gene dosage or positional effects from genes that are in cis with families of SDs. In array CGH studies, breakpoint definition and determination of copy number changes within SDs in genomic disorders may be confounded by rearrangements within nearly identical low copy sequences. Angelman and Prader-Willi (AS, PWS) syndromes are examples of a meiotically unstable deletion on chromosome 15q11-q13 flanked by complex SDs. The deletion involves a large Class I and a small Class II rearrangement sharing common telomeric breakage. We have developed a FISH strategy aimed at localizing rearrangements within these SDs. Sequence-based single (sc) and low copy (lc) genomic probes are differentially labeled and used individually or in combination to determine contextual copy number changes within specific duplicon family members. Widely separated lc probes $(>3$ $\mathrm{Mb})$ are distinguishable as multiplex hybridizations. We have designed and validated $47 \mathrm{sc}$ and lc probe sequences which give context of duplicon families in situ on metaphase chromosomes and are using these reagents to analyze deletions in AS patients. The intervals occurring at the interfaces of greatest breakage activity were used to develop and validate FISH probes $1.5-5 \mathrm{~kb}$ in length. In situ hybridization algorithms were used to accurately localize proximate regions of SD blocks where breaks are more likely for Class I and Class II AS patients. The studies delineate inter-patient variability of segment sizes at both the proximal and distal ends. Greater precision and granularity can be obtained by aggregating additional single copy and low copy probes in regions of SDs that have been systematically narrowed, expediting breakpoint determination. Ultimately, this approach can delineate the sites of interruption in genes mapped to SD regions, and provide insight into mechanisms by which SD-mediated deletions arise.

\section{8 \\ Analysis of Products of Conception by Microarray Analysis \\ J.L. Smith ${ }^{\text {a }}$ S. Schwartz ${ }^{\text {b }}$, J. Tepperberg ${ }^{\text {b }}$, P.H. Papenhausen ${ }^{\text {b }}$ \\ a Laboratory Corporation of America/Dynagene, Houston, Tex., bLaboratory Corporation of America, Research Triangle Park, N.C., USA}

Over $50 \%$ of first trimester pregnancy losses are chromosomally abnormal; while the majority of abnormalities are numerical and de novo, an explanation for the loss is important. Culturing tissue for cytogenetic analysis from products of conception can be problematic. Bacterial contamination secondary to non-sterile collection and low fetal tissue viability can result in culture failure; overgrowth of fetal cells by maternal cells can leave results in question. Utility of the Affymetrix SNP microarray analysis of POC tissues was tested to determine if this technology could increase diagnostic yield.

Analysis of 32 POCs using the 6.0 microarray was performed on cultured cells (11 cases) and on direct tissue (21 samples). All arrays in the cultured analysis were concordant with cytogenetics, including 6 trisomies and 1 triploid. The dosage of abnormal tissue to normal maternal tissue was easily estimated. In abnormal or male DNA mixes, percentages of normal female cells were usually higher by cytogenetics than by array estimation, suggesting a maternal cell proliferative advantage in vitro.

Direct tissue DNA extraction was performed on residual tissue (stored at $4^{\circ} \mathrm{C}$ ) 1-6 weeks after original culture set-up. Seventeen samples had failed to grow in culture. Despite extended storage time, all arrays yielded high quality results with 5 aneuploid, 10 normal male and 6 normal female. Four samples were studied because of previous normal female cytogenetic results with possible maternal cell overgrowth. Although DNA isolation was from tissue previously dissected of tissue most likely to be fetal, one conclusively showed a male with trisomy 21 and 22 .

SNP microarrays have a major advantage over CGH-based arrays as allele ratios from SNP analysis can identify tissue mixes and diagnose triploidy. In both array types, dosage is 'normalized' by software analysis; however, the 2:1 heterozygote allele ratio for all chromosomes in the SNP microarray provides the triploid diagnosis. This same ratio provides evidence of normal female fetal cells in maternal tissue. The lowest threshold for detection of fetal tissue was not tested, but 2 cases with $<10 \%$ mosaicism have been detected in blood. These data show the efficacy of a genotyping microarray platform for analysis of POCs.

\section{9 \\ Development of LOINC for Integrating Constitutional Cytogenetic Test Result Reports into Electronic Medical Record \\ Y. Heras ${ }^{\mathrm{a}, \mathrm{b}}$, A.R.Brothman ', M.S. Williams a, J.A. Mitchell ${ }^{\mathrm{b}}$, S.M. Huff ${ }^{\mathrm{a}, \mathrm{b}}$ \\ antermountain Healthcare, ${ }^{b}$ Department of Biomedical Informatics, 'Departments of Pediatrics, Human Genetics and Pathology, University of Utah, and ARUP Laboratories, Salt Lake City, Utah, USA}

Objective: To develop Logical Observation Identifiers Names and Codes (LOINC) codes to represent constitutional cytogenetic test names and results for electronically exchanging coded and structured result reports. The LOINC codes developed must be flexible and sustainable for easy maintenance. The goal is to create a standard set of codes that are flexible enough to be used for all unique conventional and molecular cytogenetic results.

Background: Integration of genetic and genomic data with traditional clinical data to support diagnostic and treatment reflects a new era of personalized medicine. This depends on effective communication between laboratories and clinicians, and on ef-

Preprint Cytogenet Genome Res 128/4/2010

Abstracts will be available online, free of charge 
fective and timely access to the right information. Electronic Medical Records (EMRs) promise to improve patient care by providing advanced Clinical Decision Support (CDS) at the point of care. Standard terminologies that are tightly coupled with standard information models are the foundations of developing CDS enabled EMRs. Current standard terminologies are not sufficient to encode cytogenetic test results.

Design: Patient de-identified sample result reports were obtained from ARUP Laboratories for a variety of normal and abnormal constitutional studies using G-banding, FISH and array $\mathrm{CGH}$. Information models were created to capture the semantic relationships of the key data elements that existed in the reports. Sample reports were subsequently obtained from Emory and Mayo Clinic Cytogenetics Laboratories to verify the information models. The information models were then used to guide the systematic creation of the LOINC codes.

Results: Three information models were created and will be presented. Fifty-three unique LOINC codes were proposed based on the information models. A post-coordinated approach was used in developing these LOINC codes. LOINC panel codes were created to represent the hierarchical structures implied by the reports.

Conclusion: The proposed LOINC codes met our objective and will allow the use of well established health informatics standards to exchange coded and structured cytogenetic test results between testing laboratories and ordering institutions. Use of standard structures and terminologies for cytogenetic results is critical for effective communication between testing laboratories and clinicians. This minimizes misinterpretation, leads to consistency, and provides the EMR systems flexibility of customizing formatting to present more clinician-friendly reports.

\section{0}

\section{SNP Microarray Detects Mosaic Segmental Uniparental Isodisomy in Three Cases}

T.A. Smolarek, K. Lipscomb Sund, H.M. Saal, R. Hopkin, L. Bao, S.L. Zimmerman

Cincinnati Children's Hospital Medical Center, Division of Human Genetics, University of Cincinnati, Department of Pediatrics, Cincinnati, Ohio, USA

DNA microarray studies are useful for the identification of pathogenic changes and for clarification of breakpoints in patients with previously known chromosome abnormalities. SNP microarray also will detect uniparental isodisomy (stretches of homozygosity for alleles or copy neutral loss of heterozygosity), which may provide additional information about a patient's phenotype.

DNA was analyzed from patients with different phenotypes using the Illumina 610K SNP array to detect clinically significant DNA copy number changes or to better characterize known chromosome abnormalities. Three patients were identified with mosaicism for extensive stretches of homozygosity for alleles ranging in size from $67 \mathrm{Mb}-148 \mathrm{Mb}$. The first was a female patient with a known 1p36 deletion referred for better definition of the breakpoints due to a milder clinical presentation. In addition to the breakpoint characterization, we identified an unexpected pattern of extensive homozygosity of alleles for most of $1 \mathrm{p}$. The B allele frequency plot suggested either a low level mosaic duplication for most of $1 \mathrm{p}(1 \mathrm{p} 36.32 \rightarrow \mathrm{p} 13.3)$ or mosaicism for uniparental isodisomy. Follow-up studies of metaphase and interphase cells using fluorescence in situ hybridization (FISH) showed no evidence of trisomy for $1 \mathrm{p}$ ruling out mosaic duplication and suggested mosaic segmental uniparental isodisomy. The second patient was referred with cleft lip, behavior problems, mild mental retardation, attention deficit disorder, bipolar disorder and learning difficulties. No clinically significant DNA copy number changes were identified. A similar pattern was identified on the B allele frequency plot for 11q (11q13.2 $\rightarrow$ q25). FISH results showed no DNA copy number changes for 11q. The third patient presented with an indication of a suspected immunodeficiency syndrome. The B allele frequency plot showed mosaic uniparental isodisomy for $2 \mathrm{q}$ $(2 \mathrm{q} 11.1 \rightarrow \mathrm{q} 37.3)$.

SNP microarray technology has been useful in highlighting additional genomic complexity in patients with an abnormal phenotype. These findings may contribute to some of the phenotypic variability identified in patients with the identical chromosome abnormality. It is unknown whether mosaicism for segmental uniparental isodisomy contributed to the phenotypes of the patients in this study. Therefore, future studies will help delineate the clinical significance of segmental uniparental isodisomy and its association with a patient's phenotype.

\section{1 \\ An Act of Generosity Turned into a Quandary by Unexpected Mosaicism: Case Report of Constitutional Trisomy 8 Mosaicism in a Healthy Bone Marrow Donor}

N. Uddin ${ }^{\mathrm{a}-\mathrm{c}}$, M.S. Williams ${ }^{\mathrm{a}}$, S.T. South ${ }^{\mathrm{a}-\mathrm{c}}$

Departments of apediatrics and ' ${ }^{b}$ Pathology, University of Utah, and 'ARUP Laboratories, Salt Lake City, Utah, USA

Incidental identification of constitutional chromosomal aberrations in bone marrow donors is occasionally documented in the literature. Here we present, for the first time, a case of incidental identification of constitutional trisomy 8 mosaicism (CT8M) in a healthy marrow donor who was identified after the recipient developed donor derived trisomy 8 (T8), post-transplant.

The donor, a 29-year-old female was referred for evaluation of possible CT8M. She had normal pre-donation peripheral blood counts, no significant medical history and had 3 normal children. She donated for a same gender, matched unrelated recipient who had acute myeloid leukemia with 11q23/MLL rearrangement but without $\mathrm{T} 8$ in the neoplastic clone. The recipient acquired marrow T8 after complete marrow engraftment with no evidence of recurrent disease or development of a new neoplasm. The donor had normal intelligence, normal physical examination except a subtle pigmentary abnormality of the right upper arm. For cytogenetic evaluation of the donor, chromosome analyses on peripheral blood and skin fibroblasts and FISH with chromosome 8 probe (CEP8, Abbott Molecular) on peripheral blood was performed. It revealed CT8M in the donor with disproportionate tissue involvement (20-36\% trisomy in blood, 
$\sim 2 \%$ trisomy in fibroblasts). The karyotype and FISH ISCN was described as follows:

Blood: 47,XX,+8[4]/46,XX[16]

Blood: nuc ish 8 cen $(D 8 Z 2 \times 3)[72 / 200]$

Skin fibroblasts: 47,XX,+8[1]/46,XX[50]

Salient features of CT8M include facial dysmorphism, mild to moderate mental retardation and skeletal abnormalities. However clinical features can be very variable and mild. No good correlation exists between the extent and severity of clinical manifestations and mosaicism level. Increased risk of spontaneous abortions is possible but as trisomy 8 is believed to be mostly a postzygotic event, increased risk of trisomy 8 mosaicism in the progeny seems unlikely. There is somewhat inconclusive evidence for increased risk of neoplasia (especially hematological) with CT8M. Association of CT8M with Bechet Disease has also been reported.

The diagnosis of CT8M in the donor was completely unexpected and posed a unique management dilemma for a healthy female of reproductive age. Because of paucity of data, no conclusions can be drawn for the neoplastic risk in patients with CT8M therefore only close follow-up was suggested.

\section{2 \\ Detection of Cytogenetic Abnormalities by Fluorescent in situ Hybridization (FISH) after Isolation of Plasma Cells}

\section{H. Aviv, I. Maxwell, C. Creese, K. Vassallo}

Robert Wood Johnson Medical School and Robert Wood Johnson University Hospital, New Brunswick, N.J., USA

Plasma cell neoplasms result from clonal expansion of terminally differentiated B-cells. The diagnosis of plasma cell myeloma is made when plasma cells comprise at least $30 \%$ of the bone marrow volume. CD138 stain is useful for quantifying plasma cells, and clonality is established with stains for kappa and lambda light chains. The number of plasma cells seen in the bone marrow aspirate varies from barely increased to upwards of $90 \%$. The low number may be due to suboptimal aspirate or the frequent focal distribution of myeloma in the bone marrow. About $1 / 3$ of myelomas are abnormal by karyotype, and FISH increases the detection rate to $>90 \%$. However, detection of abnormalities with respect to the number of clonal plasma cells in the bone marrow aspirate has not been established.

We implemented isolation of plasma cells from the bone marrow aspirate by magnetic labeling of CD138+ cells followed by separation by positive selection. In late 2009 , we performed plasma cell isolation in 42 bone marrows. Eighteen were normal and 24 abnormal. Fifteen cases were hyperdiploid, 7 with deletion of $13 \mathrm{q}$ or $17 \mathrm{p}$. Eight cases had CCND1/IGH fusion, one with deletion of 13q. One case had only deletion $13 \mathrm{q}$ and $14 \mathrm{q}$. One case was initially hyperdiploid, but acquired CCND1/IGH fusion at a subsequent analysis. Other $I G H$ translocations were not observed. The number of plasma cells in the cytogenetically normal bone marrow aspirates ranged from 0 to $10 \%$ (mean $2.5 \%$ ). The one case with $10 \%$ of plasma cells was polyclonal. The number of plasma cells in the aspirate of the abnormal cases ranged from $0.5 \%$ to $80 \%($ mean $=17 \%$, median $=5 \%)$. Abnormalities were detected in
5 bone marrows with less than $3 \%$ of non-clonal plasma cells by flow cytometry. Abnormalities were found in 8 additional samples with fewer than $5 \%$ of clonal plasma cells. All samples with more than $5 \%$ of clonal plasma cells in the aspirate were abnormal. Thus, FISH after plasma cell isolation is a more sensitive method for detection of abnormalities in plasma cell myeloma than flow cytometry. Without this technique, abnormalities in cases with low numbers of plasma cells would not be detected.

13

\section{Apparently Novel EWSR1 Gene Rearrangements Detected in Two Astroblastomas}

K. Craddock ${ }^{\mathrm{a}}$, I. Sorokin ${ }^{\mathrm{b}}$, G. Nie ${ }^{\mathrm{b}}$, M. Zielenska ${ }^{\mathrm{b}, \mathrm{c}}$, W. Halliday ${ }^{\mathrm{b}, \mathrm{c}}$, M. Shago b, c

aDepartment of Pathology, University Health Network, ${ }^{b}$ Department of Paediatric Laboratory Medicine, The Hospital for Sick Children, 'Department of Laboratory Medicine and Pathobiology, University of Toronto, Toronto, Ont., Canada

Background: Astroblastoma is a rare supratentorial brain tumour of infants and young adults, characterized by an epithelioid cell population with prominent perivascular pseudo-rosettes, and protein expression of GFAP, S100, and EMA. In the $<10$ karyotypes reported thus far, chromosomes 7, 10, 12, 21, and 22 have been noted to be frequently involved in both structural and numerical structural changes, however, no specific genes or translocations have been previously demonstrated to be recurrently involved in these tumours. We describe for the first time the presence of EWSR 1 gene rearrangements in 2 separate astroblastomas.

Methods: FISH using a commercial break-apart probe set for the EWSR1 gene was performed on nuclei isolated from formalinfixed, paraffin-embedded tissue of 4 astroblastomas, as well as 5 ependymomas, and 5 astrocytomas, chosen for their histologic similarity to astroblastomas. For those cases positive for EWSR1 gene rearrangement by FISH, known EWSR1 fusion transcripts were tested for using reverse-transcription followed by PCR. Three of the astroblastomas had previously been cultured and karyotyped, and 1 case had been investigated by SKY.

Results: Two of the 4 astroblastomas were positive for EWSR1 gene rearrangement by FISH. None of the ependymomas or astrocytomas showed such a rearrangement. One case had a $t(10 ; 22)$ (q25;q12) with the 3' EWSR1 signal translocated to the der(10) and a loss of the corresponding $5^{\prime}$ EWSR1 signal. The other case had 2 marker chromosomes, one of which was revealed by SKY to be an $\operatorname{add}(22)(\mathrm{p}$ ?). RT-PCR testing for known fusion transcripts including EWSR1-FLI1, EWSR1-ERG, EWSR1-ETV1, EWSR1-WT1, and EWSR1-ATF1 was negative in one case that had fresh frozen material available for RNA extraction.

Discussion: We describe for the first time an EWSR1 gene rearrangement in 2 separate astroblastomas. The resulting fusion transcript is not one of those recurrently seen in Ewing sarcoma, clear cell sarcoma, or intra-abdominal desmoplastic small round cell tumour, and may represent a novel rearrangement that is present in a subset of cases that fit the histologic and immunophenotypic features of astroblastoma. Further studies to attempt to identify the fusion partner(s) in these astroblastomas are pending. 


\section{4}

Characterization of Uncommon BCR and $A B L 1$ Breakpoints in Three Cases of Chronic Myelogenous Leukemia

F.C. Wheeler ${ }^{\mathrm{a}}$, K. Kaiser-Rogers ${ }^{\mathrm{a}, \mathrm{b}}$, K. Muldrew ${ }^{\mathrm{b}}$, M.L. Gulley ${ }^{\mathrm{b}}$, K.W. $R a o^{a, b}$

${ }^{a}$ Department of Pathology and Laboratory Medicine and

bepartment of Pediatrics, University of North Carolina,

Chapel Hill, N.C., USA

We have identified 3 cases of chronic myelogenous leukemia (CML) with atypical BCR or $A B L 1$ breakpoints. CML is a myeloproliferative disorder characterized by the Philadelphia $(\mathrm{Ph})$ chromosome produced secondary to the reciprocal translocation between the $A B L 1$ and $B C R$ genes on chromosomes 9 and 22 respectively. The $B C R-A B L 1$ fusion gene produces a constitutively active tyrosine kinase that increases cell proliferation. Ninety-five percent of CML and 20-30\% of acute lymphoblastic leukemia (ALL) are characterized by the presence of the $\mathrm{Ph}$ chromosome. At the molecular level, the frequencies with which various $B C R$ breakpoints are used differ for these 2 malignancies. In $\mathrm{Ph}+$ cases, the major breakpoint $(\mathrm{M}-B C R)$ occurs in $>99 \%$ of CML and results in the p210 fusion product. The minor breakpoint (m-BCR) is associated with ALL and results in the 190 fusion product. The $\mathrm{m}-B C R$ breakpoint and the micro breakpoint $(\mu-B C R, \mathrm{p} 230 \mathrm{fu}-$ sion product) occur in $<1 \%$ of CML cases. Other variant $B C R$ and $A B L 1$ breakpoints are rare. Here we describe 3 cases of CML, 2 with atypical breakpoints and 1 with the rarely documented $\mathrm{m}$ $B C R$ breakpoint. The first patient was $\mathrm{Ph}+$ and appeared to have the $\mathrm{m}-B C R$ using $B C R-A B L 1$ ES-FISH. The second patient had a complex rearrangement involving chromosomes 1, 9 and 22, and was positive by FISH for the M- $B C R-A B L 1$ fusion. Molecular testing by Q-RT-PCR did not confirm the presence of the fusion transcript using primers for both $\mathrm{M}-B C R$ and $\mathrm{m}-B C R$ in either patient. Additional characterization revealed the reason molecular testing failed to detect a fusion transcript. Case 1 had a typical m-BCR breakpoint (E1) but an atypical $A B L 1$ breakpoint (A3), resulting in a variant p190 fusion product. Case 2 had an unusual $B C R$ breakpoint located between the $\mathrm{m}-B C R$ and $\mathrm{M}-B C R$ breakpoints (E6), resulting in a fusion product of unknown size. Case 3 is a patient with CML with the $\mathrm{m}-B C R$ breakpoint typically seen in ALL. Our experience with the patients described above indicates that Q-RT-PCR should be performed on diagnostic samples to determine the utility of molecular testing for patient monitoring. Furthermore, testing for variant breakpoints is warranted in cases of $\mathrm{Ph}+\mathrm{CML}$ without detectable $\mathrm{M}-B C R$ or $\mathrm{m}-B C R$ transcripts.

\section{5}

\section{Chromosome Kissing and Cancer}

B.L. Flynn a , E. Pizzo a J. Crivello a, b J. Brown ${ }^{\mathrm{a}, \mathrm{b}}$

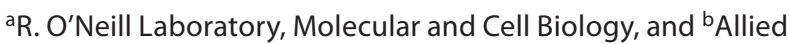

Health Sciences, University of Connecticut, Storrs, Conn., USA

Specific recurring rearrangements are present at a high frequency in cancer cells and permit the classification of a specific cancer type, crucial for the prognosis and treatment of the dis- ease. It is not completely understood why these recurring chromosome translocations happen at the same breakpoints in the genome, however it is clear that certain regions are more susceptible to instability than others. Breakpoints associated with cancer often coincide with evolutionary breakpoints, boundaries for ancestral chromosome segments. Evolutionary breakpoints occur at non-random locations in the human karyotype and contain common fragile sites or other unstable sequences. The observed co-localization of evolutionary and cancer-prone breakpoint loci begs the question; is there a functional correlation between evolutionary breakpoints and the genesis of chromosome translocations and cancer? We are determining the frequency of overlap between evolutionary breakpoints and leukemia-associated breakpoints, 'chromosome kissing', in the nuclei of differentiated and progenitor cells using fluorescence microscopy and image deconvolution. As it is not known whether cancer stem cells arise from malignant transformation of a tissue-specific stem cell, committed progenitors or differentiated cells that re-acquire stem cell-like properties, interchromosomal interactions at the breakpoint 'hot spots' are also being evaluated in both normal and malignant stem cell and differentiated cell lines. A positive association of interchromosomal interactions between evolutionary breakpoint loci and recurring cancer breakpoint loci in progenitor or stem cells may allow for the design of a targeted screening or diagnostic assay for interchromosomal interactions in specific cell lineages. The frequency of chromosome kissing in different cell types will provide fundamental insights regarding the pathological process of cancer, specifically the role of locus-specific interchromosomal interactions and the formation of chromosomal rearrangements in cancer.

\section{6 \\ Stimulation of Chronic Lymphocytic Leukemia (CLL) Cells with CpG Oligodeoxynucleotides (ODN) Gives Consistent Karyotypic Results among Laboratories: A CLL Research Consortium (CRC) ${ }^{\mathrm{h}}$ Study}

N.A. Heerema a , J.C. Byrd a , P.Dal Cin ${ }^{\text {b }}$, M.L. Dell' Aquila ${ }^{c}$, P. Koduru ', A. Aviram ', S. Smoley ', L.Z. Rassenti ', A. Greaves ', J.R.Brown ${ }^{f}$, K.R. Rai ${ }^{9}$, T.J. Kippsc ${ }^{c}$, N.E. Kay ${ }^{\mathrm{e}}$, D. van Dyke ${ }^{\mathrm{e}}$

${ }^{\mathrm{a}}$ The Ohio State University, Columbus, Ohio, ${ }^{\mathrm{b}}$ Brigham \& Women's Hospital, Boston, Mass., 'Moores Cancer Center, University of California at San Diego, San Diego, Calif., dNorth Shore Hospital, Manhasset, N.Y., 'Mayo Clinic, Rochester, Minn., fDana-Farber Cancer Institute, Boston, Mass., 9North Shore Long Island Jewish Medical Center, New Hyde Park, N.Y.,

hfor the Chronic Lymphocytic Leukemia Research Consortium, University of California at San Diego, La Jolla, Calif., USA

Background: Cytogenetic abnormalities in CLL are important prognostic indicators. Until recently, only interphase cytogenetics was clinically useful in CLL because traditional mitogens are not effective mitotic stimulants. Recently, CpG-ODN stimulation has shown effectiveness as a CLL mitogen. The reproducibility of abnormalities detected after CpG-ODN stimulation has not been examined. The CRC conducted a prospective trial to test whether (1) CpG-ODN enhances detection of abnormal clones, (2) the 
CRC cytogenetic laboratories would achieve similar results on the same samples, and (3) CpG-ODN causes cytogenetic abnormalities. Methods: One laboratory compared stimulation of CLL cells using CpG-ODN versus pokeweed mitogen (PWM) + 12-O-tetradecanoyl-phorobol-13-acetate (TPA). To test the reproducibility of CpG-ODN stimulation, fresh blood samples from 1 normal control and 12 CLL patients evaluated at UCSD were collected and distributed (blinded to phenotype) to 5 CRC cytogenetic laboratories where they were cultured for 3 days using CpG-ODN stimulation. Two laboratories did interphase FISH analyses using standard CLL probes. Results: For PWM+TPA versus CpG-ODN comparison, more cases had clonal abnormalities with CpGODN $(147 / 229,64 \%)$ than with PWM+TPA (11/229, 48\%; p = $0.0005)$. All clonal abnormalities in the PWM+TPA cultures were also observed in the CpG-ODN cultures. In the 5-laboratory CpG-ODN comparison, all karyotypic results were concordant with FISH. The control had normal results in all 5 laboratories. A minor abnormal clone was found in 3 cases in 1 or 2 labs, confirmed by FISH for two. Three cases exhibited complex karyotypes, interpretations varied, but all laboratories identified the same complex clones. All cases had nonclonal cells, with no pattern detected among the 5 labs. Conclusions: Abnormal clones in CLL are more readily detected with CpG-ODN stimulation than with traditional B-cell mitogens. The clonal abnormalities revealed by $\mathrm{CpG}$-ODN stimulation are reproducible among different cytogenetic laboratories and are consistent with the 'gold standard' interphase FISH results. CpG-ODN did not appear to create new clonal B-cell populations during 3 days of culture. CpGODN stimulation enhances detection of clonal cytogenetic abnormalities and complexity in CLL. Complexity, which cannot be determined by standard FISH analyses, is prognostic. Therefore, stimulation with CpG-ODN is useful to fully characterize cytogenetic prognostic factors in CLL.

\section{7 \\ Heterogeneous MLL Abnormalities in 509 Consecutively Newly Diagnosed Pediatric Acute Leukemias}

X.-Y. Lu, M. Folsom, M. Azim, J. Ringrose, S. Plon, C. Lau, C. Steuber, J. Margolin, K. Rabin, P. Rao

Texas Children's Hospital Cancer Center, Department of

Pediatrics, Baylor College of Medicine, Houston, Tex., USA

Chromosomal translocations leading to $M L L$ gene (11q23) rearrangements are common and associated with poor prognosis in pediatric acute leukemia. However, other MLL abnormalities such as deletions, duplications, cryptic insertion or complex inversions/insertions are also not uncommon in pediatric leukemias. To gain insight into the frequency of these distinct nonrandom $M L L$ events, we reviewed the G-banding and fluorescence in situ hybridization (FISH) data from 509 consecutive pediatric de novo acute leukemia cases consisting of $430 \mathrm{~B}$-cell precursor acute lymphoblastic leukemia (BCP-ALL) and 79 acute myeloid leukemia (AML) samples that were tested in our clinical cytogenetics laboratory at Texas Children's Hospital, Baylor College of Medicine. MLL abnormalities were identified in $7.4 \%$ $(32 / 420)$ of the BCP-ALLs and $18.9 \%(15 / 79)$ of the AMLs. Of these, $M L L$ genes involving common recurrent translocations e.g. $\mathrm{t}(4 ; 11), \mathrm{t}(11 ; 19), \mathrm{t}(6 ; 11)$, and $\mathrm{t}(9 ; 11)$ were found in 24 cases, and other $M L L$ abnormalities were identified in the remaining 23 cases. Cryptic 5' $M L L$ insertion, masked by apparent abnormal karyotypes, was revealed by $M L L$ metaphase FISH in 4 cases including one ins $(4 ; 11)$, one ins $(9 ; 11)$ and two ins $(10 ; 11)$. Additionally, complex $M L L$ inversion-translocation involving bands 11q13-q23 was detected by metaphase FISH in 3 cases with apparent $t(10 ; 11)$ and 1 case apparent with $t(1 ; 11)$ translocation. Copy number aberrations involving the entire $M L L$ gene were identified in 10 cases. Of which, 8 BCP-ALL cases showed loss of entire $M L L$ and the remaining 2 AML cases displayed gain of entire $M L L$ resulting from unbalanced $t(7 ; 11)$ translocations. Interestingly, other chromosomal aberrations were also identified in the $M L L$ deleted BCP-ALL cases, notably with ETV6-RUNX1 fusion (n = $3)$, increased copy number of RUNX1 ( $\mathrm{n}=3)$ and deletion of ETV6 $(\mathrm{n}=1)$. Our data suggest that atypical $M L L$ rearrangements are frequent and metaphase FISH plays an important role in defining these hidden $M L L$ rearrangements. The $M L L$ gene deletion exclusively identified in BCP-ALL in this study had strong correlation with loss of ETV6, gain of RUNX1 or ETV6-RUNX1 fusion and may represent a specific cytogenetic subgroup.

\section{8 \\ Molecular Study of Complex Karyotypes in Bone Marrow Cells of Patients with Myelodysplastic Syndromes (MDS)}
Z. Zemanova a , K. Michalova a, b, J. Brezinova ${ }^{\text {b }}$ L. Lizcova $^{\text {a }}$, S. Izakova ${ }^{\text {b }, ~ D . ~ B y s t r i c k a ~}{ }^{\text {a }}$ I. Sarova ${ }^{\text {b }}$, M. Siskova ${ }^{\text {c }}$, O. Cerna ${ }^{\text {d, }}$ J. Cermak ${ }^{b}$
${ }^{a}$ Center of Oncocytogenetics, Institute of Clinical Biochemistry and Laboratory Diagnostics, General Teaching Hospital and First Faculty of Medicine, Charles University in Prague, bInstitute of Hematology and Blood Transfusion, ${ }^{c} 1$ st Medical Department, General Teaching Hospital and First Faculty of Medicine, Charles University in Prague, dDepartment of Clinical Hematology, Faculty Hospital Kralovske Vinohrady, Prague, Czech Republic

Complex chromosomal aberrations (CCA) are in most studies defined as 3 or more chromosomal changes in one karyotype. CCA are present in bone marrow of $10-20 \%$ patients with MDS and are associated with treatment resistance and poor prognosis. In this study various modifications of molecular cytogenetic techniques were used to analyze chromosomal regions and breakpoints involved in CCA: FISH with locus-specific probes (Abbott Vysis), CGH, mFISH/mBAND (MetaSystems) and/or array CGH (BlueGnome, NimbleGene) for which DNA was prepared by amplification from cells fixed for cytogenetic analyses.

During 2002-2008 bone marrow of 680 adults at diagnosis of MDS was examined and in 86 of them (12.6\%, primary MDS was diagnosed in 74, secondary in 12 patients) CCA were ascertained. Deletion of $5 \mathrm{q} 31$ region was proved in 75 patients $(87.2 \%), \mathrm{mFISH}$ analyses in 35 cases showed that parts of deleted No. 5 were translocated into other chromosomes. Translocations were non-balanced and recurrent partners were chromosomes $17(11 \times), 3$ $(6 \times), 7(5 \times)$ and $12(5 \times)$. Monosomy of chromosome 5 was con-

Preprint Cytogenet Genome Res 128/4/2010

Abstracts will be available online, free of charge 
firmed in 1 case only by mFISH, however by arrayCGH translocated part of short arm of deleted 5 was identified. It can be presumed that -5 is not a separate diagnostic entity in MDS as quoted in theliterature. The most frequent breakpoints on chromosome 5 determined by mBAND were $5 q 33(31 \times), 5 q 13(25 \times), 5 q 14(7 \times)$ and $5 \mathrm{q} 12(5 \times)$. Besides chromosome 5 , mostly involved in CCA were chromosomes $7(37 \times), 3(33 \times), 12(31 \times), 17(31 \times)$ and 11 $(24 \times)$, the most recurrent breakpoints were at regions $7 \mathrm{p} 11(6 \times)$, $7 \mathrm{q} 11(6 \times)$ and $12 \mathrm{p} 11(7 \times)$. CCA in bone marrow at diagnosis was in this cohort accompanied by poor response to therapy and short survival (median 5 months).

Using combination of molecular cytogenetic techniques a wide variety of cryptic aberrations not detectable by conventional cytogenetics were found. No new recurrent rearrangement was found. Non-random and frequent breakpoints precisely identified by molecular techniques might lead to discovery of genes, involved not only in origin but also in progress of malignancy and to better understanding of molecular etiology of MDS.

Supported by: MZOVFN2005, MSM0021620808, MSMTLC535, COST-EUGESMA, IGAMZCR NR/9227-3.

\section{9 \\ Emerging Data Using a Targeted Oligo-Based Microarray in the Myelodysplastic Syndromes Reveal Provocative Complexity and New Recurrent Genetic Aberrations}

M.L. Slovak a , T. Brown ${ }^{\text {b , J.-Y. Han }}{ }^{\text {c }, \text { L.J. Campbell }}{ }^{\text {, }}$ M. Wall ${ }^{\text {, }}$, L.GShaffer ${ }^{\text {a }, B . B a l l i f}{ }^{\text {a }}$, R. Schultz ${ }^{\text {a }}$

Signature Genomics, Spokane, Wash., USA

Background: The myelodysplastic syndromes (MDS) are a heterogeneous group of clonal disorders characterized by ineffective hematopoiesis, cytopenias, and a risk of transformation to acute myeloid leukemia (AML). With the advent of targeted therapeutic strategies in MDS, selecting the appropriate therapy for each individual patient is critical to optimize clinical benefit. To address this concern, we analyzed blood or bone marrow MDS samples by microarray-based comparative genomic hybridization (aCGH) using a high-resolution, targeted oligo-based microarray. Experimental design: 36 newly-diagnosed adult MDS samples were obtained from 3 centers from August-December 2009. The aCGH data (gains and losses) were compared to their corresponding conventional cytogenetics and FISH. Cryptic aberrations $(\sim 5$ $\mathrm{Mb}$ ) were confirmed by FISH analysis. Results: Copy number aberrations (CNAs) (range 0-31, median 5) were identified by aCGH in 35/36 (97\%) samples. Seven non-complex cases (1-2 CNAs) showed identical cytogenetics and aCGH results; 23 samples showed additional CNAs by aCGH. aCGH revealed new cytogenetic aberrations in 22/36 ( 60\%) patients and refined complex aberrations in $18 / 36(50 \%)$. Cryptic aberrations ( $\leq 5 \mathrm{Mb})$ were identified in 21 cases $(\sim 60 \%)$ and included genetic alterations previously associated with MDS/MPD oncogenesis such as deletions of NF1 (4), RUNX1 (3), RASSF1 (2), CCND1 (2), MYC (2), and 1 case each with deletion of TET2, JUN1, DNMT3A, HRAS, PDGFRA, or FIP1L1. An unusual 500-kb 5q35.3 deletion was detected in 1 sample and another case showed an internal FLT3 duplication. Gene amplification was observed in 4 cases (11\%) and included CUL7/6p21.1, ETS/11q24.3, DNMT1/19p13.2, and ERG/ ETS/21q22.3. Complete or partial discordant calls were found in samples carrying balanced translocations or inversions $(n=4)$, the presence of multiple clones with a low-level clone not detected $(\mathrm{n}=1)$, or bone marrow involvement below the limits of aCGH sensitivity $(<20 \%)(\mathrm{n}=2)$. Enrichment techniques are suggested to improve detection of less frequent clones, especially in patients with low-grade MDS. Conclusion: Our preliminary oligo aCGH results show the potential of this technology to identify common and uncommon or cryptic recurring imbalances in MDS that may prove to be significant in disease progression, transformation to AML or improve the suitability or efficacy of molecularly targeted therapy.

20

\section{Implementation of a High Density SNP Array with Cytogenetic and Molecular Techniques for the Characterization of Genetic Aberrations in a Patient with CML and Resistance to Imatinib Mesylate}

R. Toydemir a, b , L. Rowe ${ }^{c}$, M. Hibbard ${ }^{\mathrm{d}}$, M. Salama ${ }^{\mathrm{a}}$, b S. Shetty ${ }^{\mathrm{a}, \mathrm{b}}$

aDepartment of Pathology, University of Utah, ${ }^{\mathrm{b}}$ ARUP

Laboratories, ' $A R U P$ Institute for Clinical and Experimental

Pathology, Salt Lake City, Utah, ${ }^{\mathrm{d} C l a r i e n t, ~ I n c ., ~ A l i s o ~ V i e j o, ~ C a l i f ., ~}$ USA

New molecular technologies, including high density SNP arrays, are being used more frequently in diagnostic laboratories. These technologies can provide genetic information beyond the limits of conventional cytogenetic studies. We present a case where a high density SNP array was used in combination with chromosome studies, FISH, and sequence analysis, where each technique provided valuable information to understand the clinical presentation of this patient.

The patient was a 36-year-old male, referred for chromosome studies with history of CML and no response to imatinib mesylate. Chromosome analysis showed an inversion within the long arm of both homologues of chromosome 3 and an interstitial deletion within the long arm of one chromosome 7. The rearrangement of EVI1 locus on both homologues of chromosome 3 was confirmed by FISH. Additional FISH studies showed a cryptic insertion of $A B L 1$ into $B C R$ region, and subsequent duplication of the Philadelphia chromosome. Analysis of the $B C R-A B L$ fusion transcript by cDNA sequencing revealed a missense mutation in the tyrosine kinase domain, which is known to be associated with resistance to imatinib mesylate.

Rearrangements of chromosome 3 involving bands $3 q 21$ and 3 q 26 have been reported in $\sim 2 \%$ of patients with AML, and rarely in myelodysplastic syndrome or CML. To date, only 5 cases of inversion of both homologues have been reported. Loss of normal chromosome 3 and duplication of the inverted chromosome have been proposed as the most likely mechanism. We tested this hypothesis by determining the copy-neutral loss-of-heterozygosity $(\mathrm{CN}-\mathrm{LOH})$ with a SNP-array. The SNP-array showed CN-LOH on 
chromosome 3 q21-qter suggesting a somatic repair mechanism is involved in the evolution of this genetic alteration. However, CNLOH was also observed on chromosome 22 as well as chromosome 14. Duplication of chromosome 22 was expected based on our $A B L-B C R$ FISH results, however, the significance of $\mathrm{CN}-\mathrm{LOH}$ of chromosome 14 remains unknown.

This case illustrates the complexity of genetic aberrations in neoplastic cells, and the value of array technology, used in concert with conventional cytogenetic methods, for a better understanding of the pathogenesis. Future studies are needed to test the utility and limits of this technology in clinical management of cancer patients.

\section{1 \\ Defining the Molecular Cytogenetic Landscape of Cancer Using Array-Based Technologies}

\section{N. Nowak}

DNA Microarray and Genomics Facility, Roswell Park Cancer Institute, Buffalo, N.Y., USA

Technologies to identify chromosomal aberrations have rapidly evolved from the latter part of the last century with the ability to visualize in a genome-wide manner alterations on chromosomal bands on metaphases to detection of nucleotide level changes on DNA based microarrays. These alterations are detected using either microarray-based CGH (Comparative Genomic Hybridization) or CNV/SNP (Copy Number Variant/Single Nucleotide Variant Polymorphism) arrays. Unlike constitutional disorders, cancer samples exhibit significant complexity that is reflected as heterogeneity resulting from normal cell contamination as well as different evolving populations of cells within a tumor. We have applied aCGH and CNV/SNP arrays as well as other integrated microarray and molecular assays to study phenotypically interesting solid tumors and leukemias in an effort to develop assays with clinical utility. A cohort of 395 patients with head and neck SCC and complete clinical follow-up was used to construct a tissue microarray (TMA). One-hundred and fifty of these patients were represented in the cohort utilized for copy number studies. Of the 395 patients in the TMA cohort 216 had evidence of cancer on follow-up with 168 confirmed deaths as a result of their cancer (follow-up average 24.5 months, median 16.5 months). Of the 179 patients with no evidence of cancer there was an average follow-up of 62.4 months (median 56 months). Of the 150 patients represented in the head and neck SCC study cohort many different comparisons were available including:

$\mathrm{pN} 0$ versus $\mathrm{pN} 1$ or greater

pT1 or pT2 versus pT3 or pT4

pN0 pT1 or pT2 versus pT3 or pT4

$\mathrm{pN} 1$ or greater $\mathrm{pT} 1$ or $\mathrm{pT} 2$ versus $\mathrm{pN} 0 \mathrm{pT} 3$ or $\mathrm{pT} 4$

pN1 or greater with no evidence of cancer FU $>47$ months versus $\mathrm{pN} 1$ or greater with evidence of cancer.

These comparisons have allowed us to make some important clinical observations in a large cohort of patients which we are currently validating. We have also performed similar studies on a defined set of leukemias with specific cytogenetic translocations in an effort to identify additional alterations and consequences of these rearrangements.
22

Genomic Alterations in Breast Cancer Cell Lines and Mouse Xenograft Models from Fresh Human Tumors

M. Herrler ${ }^{\text {a }}$, W. Ke ${ }^{\text {a }}$, J. Stanchfield ${ }^{\mathrm{a}}$, H. Zhang ${ }^{\mathrm{b}}$, G. Deng ${ }^{\mathrm{b}}$, S.S. Jeffrey ${ }^{\mathrm{b}}$

${ }^{a}$ SciGene, Sunnyvale, bStanford University School of Medicine, Stanford, Calif., USA

Currently, the sample preparation workflow for aCGH tests is cumbersome leading to errors that confound clinical results and increase re-test rates and costs. To overcome this problem, we have developed an aCGH Workstation that automates the bench work from DNA labeling to array scanning. A robotic liquid handling and incubation system (ArrayPrep ${ }^{\circledR}$ Target Preparation System) automates the labeling and magnetic bead purification of 8-96 gDNA samples, then loads them onto microarrays for hybridization using a rotating incubation system (Mai Tai ${ }^{\circledR}$ Hybridization System). Arrays are then post-processed using a robotic instrument (Little Dipper ${ }^{\circledR}$ Processor) for washing and drying. Previous studies have shown that the aCGH Workstation generates high quality labeled gDNA and highly reproducible array data from batches of 8-96 samples. It requires less than $1 \mathrm{~h}$ handson technician time and can substantially enhance test reproducibility and lower cost [1].

In this study, we have analyzed genomic alterations in breast cancer cell lines $[2,3]$ and mouse xenograft models from fresh human tumors. Clinical samples were obtained from the operating room and implanted in mammary fat pads of immunocompromised mice and tumors compared. For each sample, replicate gDNA samples were processed using the aCGH workstation and Agilent $44 \mathrm{~K}$ feature microarrays designed for genome-wide DNA copy number variation (CNV) profiling. Samples processed on the aCGH Workstation reproducibly detected complex genomic alterations in different types of tumors.

In summary, we have shown the successful automation of the aCGH laboratory workflow for detection of genetic abnormalities at high resolution in breast cancer cell lines and mouse xenograft models. The automated platform holds the potential to significantly improve test reliability and lower the cost of routine genetic analysis of clinical samples.

\section{References}

1 Herrler M, Ke W, Stanchfield J (2009). ASHG 59 ${ }^{\text {th }}$ Annual Meeting, Honolulu, Hawaii, Abstract 1442/W.

2 Pollack JR, Sørlie T, Perou CM, Rees CA, Jeffrey SS, et al. (2002). Proc Natl Acad Sci USA 99:12963-12968.

3 Shadeo A, Lam WL (2006). Breast Cancer Research 8:R9. 


\section{3}

Implementation of High Resolution SNP Array Analysis in the Clinical Cancer Cytogenetics Laboratory

J. Biegel ${ }^{\mathrm{a}-\mathrm{c}}$, D. Wilmoth ${ }^{\mathrm{b}}$, L. Wainwright $^{\mathrm{b}}$, L. Tooke $^{\mathrm{b}}$, M. Dougherty ${ }^{\text {a }}$

Departments of aPediatrics and 'Pathology, The Children's Hospital of Philadelphia, and 'University of Pennsylvania School of Medicine, Philadelphia, Pa., USA

High-resolution SNP array analysis using the Illumina $610 \mathrm{~K}$ BeadChip was incorporated into the test menu of our clinical cancer cytogenetics laboratory in July, 2008. Validation of the platform was accomplished by analysis of 150 bone marrow and 175 solid tumor specimens. Reporting criteria were selected based on the validation data. At present, we report copy number abnormalities (CNAs) with a minimum of 20 SNPs, and copy number neutral loss of heterozygosity $(\mathrm{CN}-\mathrm{LOH})$ that covers $5 \mathrm{Mb}$. The sample must contain $>20 \%$ tumor to be interpreted with confidence.

In the first 18 months, 100 bone marrows were evaluated by standard cytogenetics and/or FISH, and SNP array. Karyotypes were unavailable for $10 \%$ of cases, of which $3 \%$ demonstrated clinically significant CNAs or CN-LOH. Seven percent of the samples were concordant for karyotypes and array, whereas $4 \%$ of cases had abnormal karyotypes and normal array studies. Notable examples included balanced 11q23 MLL translocations. Approximately $25 \%$ of the leukemias demonstrated clinically significant CNAs or CN-LOH that were not detected by karyotype analysis, the most frequent of which involved losses of 9p. Several leukemias contained related clones with a variety of structural and/or numerical abnormalities that were missed by the array studies.

We also tested 100 solid tumors. Thirty percent of cases yielded clinically significant CNAs or regions of CN-LOH by array analysis that were not detected by cytogenetic studies. In contrast, $3 \%$ of cases had abnormal clones observed by karyotype analysis that were not visible on the array, all of which were balanced translocations. The ability to detect CN-LOH had the greatest impact for patients with solid tumors. Notable examples included $\mathrm{CN}-\mathrm{LOH}$ of all homologues (doubling of a haploid genome) in germ cell tumors. The solid tumor studies also unveiled underlying constitutional abnormalities that may have predisposed patients to cancer, including the identification of CN-LOH in $11 \mathrm{p} 15$ in patients with Wilms tumor.

We suggest that SNP array analysis provides complementary data to the karyotype for evaluation of bone marrow specimens. In contrast, SNP array analysis should be the initial test ordered for solid tumors.

\section{4 \\ High Resolution Genome-Wide Chromosomal Microarray Analyses of Matched Tumor and Germline DNA in Multiple Myeloma}

\section{S. Kulkarni, V. Hucthagowder, M. Evenson, C. Mullins, M. Fiala,} M. Tomasson, R. Vij

Cytogenomics and Molecular Pathology, Department of Pathology and Immunology, Washington University School of Medicine, St. Louis, Mo., USA

Chromosomal aberrations and genomic copy number alterations are detected in nearly all multiple myeloma (MM) patients. The molecular events involved in MM development have been studied using classical cytogenetics, fluorescence in situ hybridization (FISH), comparative genomic hybridization, and array comparative genomic hybridization (aCGH). Published approaches to identify global genomic changes in MM have relied solely on the high-throughput genomic scans on plasma cells selected using CD138 magnetic separations. However, this approach of studying tumor sample alone is inherently limited due to inability to carefully distinguish germline non-tumor genomic alterations with tumor-specific acquired gene changes. We performed genome-wide analysis using high-density single nucleotide polymorphism (SNP) arrays (Affymetrix SNP 6.0) to detect copy number alterations and loss of heterozygosity $(\mathrm{LOH})$ in CD138+ malignant MM cells and matched germline (skin) specimens from 11 newly diagnosed patients. To estimate the extent of genomic gains or loss in each patient, we utilized 2 control data sets. The first data set of genotyping and copy number calls on 270 control specimens was from the International HapMap project and the second in-house data set was generated from 100 normal individuals. For detection of changes in copy number and $\mathrm{LOH}$, we set a threshold of 25 or more consecutive markers showing consensus for gain or loss spanning at least a $50-\mathrm{kb}$ region. All the MM patients analyzed revealed deletions and amplifications. 10 out of 11 patients harbored extensive genomic alterations spanning the entire length of the chromosome, of which recurrent amplifications in 1q, 3, 7, 9, 11q, 15, 19, 21q, Yp and deletions in 1p, $8 \mathrm{p}, 13, \mathrm{X}$ were observed in 3 or more individuals. $\mathrm{LOH}$ ranging from $5.5 \mathrm{Mb}$ to as large as the entire $\mathrm{p}$ arm of chromosome 1 were observed in myeloma patients. To our knowledge, this is the first ultra high-density chromosomal microarray study in paired tumor and germline samples to characterize global genomic alteration in multiple myeloma. These findings demonstrate the power, accessibility and adaptability of molecular karyotyping in cancer to identify novel acquired recurrent genetic alterations that contribute to susceptibility, transformation, and response to chemotherapy. 


\section{5}

From Chromosomes to FISH to aCGH: Genomic Approaches to Study Chronic Lymphocytic Leukemia

Y.J. Liu

University of Washington, Seattle, Wash., USA

Chronic lymphocytic leukemia (CLL) is an abnormal neoplastic proliferation of $\mathrm{B}$ cells which mainly accumulate in the bone marrow and blood. Rates in CLL show significant population variation with the highest rates in the US and Europe and the lowest rates in Asia. There are about 15,000 newly diagnosed patients each year in the US. Patients with early stage CLL have a highly variable clinical course. Survival varies from 5 years to more than 25 years. Chromosomal abnormalities, examined using iFISH and karyotype, are very useful prognostic markers in clinics for risk stratification. However, due to limited targets with iFISH panel, iFISH analysis alone underestimates the complexity of chromosomal aberrations in CLL. With FISH and karyotype analysis, it is hard to map the breakpoints precisely to know the genes in the deleted region. And the knowledge of genomic structure around breakpoints will help us understand the mechanism of aberrations. Since most chromosomal abnormalities in CLL are unbalanced, they are suitable for CGH analysis. In addition, there are several advantages: CGH does not require dividing B-cells, and it is a whole genome analysis to give a more complete view of the genome and immediate knowledge of gene content in the abnormal region. For the last 10 years, our cytogenetics laboratory processed more than 1,000 CLL cases. The karyotype and FISH analyses of these samples will be presented. Array CGH results of CLL samples and its utility will be discussed. The possibility that certain population specific constitutional CNVs may contribute to CLL susceptibility is explored.

\section{6 \\ Interpretation of Microarray Results: A Point System to Discriminate Benign, Unknown Significance and Pathogenic Copy Number Changes}

\author{
D.A.S. Batista ${ }^{\text {a-c }}$, E. Wohler ${ }^{\text {a }}$, S. Morsey ${ }^{\text {a }}$, J. Biscoe a , L. Wo ${ }^{\text {a }}$ \\ N. Jinawath ${ }^{c}$ \\ ${ }^{a}$ Cytogenetics Laboratory, Kennedy Krieger Institute, \\ ${ }^{b}$ Department of Pathology, Johns Hopkins University, \\ Institute of Genetic Medicine, Johns Hopkins Medical \\ Institutions, Baltimore, Md., USA
}

Mental retardation occurs in 1-3\% of individuals in the population and about $40 \%$ of patients remain without a causative diagnosis. The use of comparative genomic hybridization and single nucleotide polymorphism (SNP) arrays has provided an answer for many of these previously undiagnosed individuals. Alongside known causative microdeletions and microduplications, many other copy number changes (CNC) of unknown clinical significance are also found. Interpretation of these CNCs is not standardized amongst cytogenetics laboratories and discordance has been previously reported to the point that some CNCs might be classified as abnormal by one laboratory and normal by another. These discrepancies highlight the need for clear and specific guidelines.

We developed a scoring system for CNCs that included 12 categories, each assigned 2, 1 or 0 points depending on the inferred likelihood of causative effect. Categories were: size and number of markers, deletion versus duplication, probe content (SNP versus cnvi), presence/absence of RefSeq genes, involvement of exons, presence/absence of microRNA, entry on OMIM, entry on DECIPHER, known syndrome, entry on CHOP CNV database of healthy individuals, entry on our laboratory internal database of benign variants and entry on DGV. The system was applied to 17 patients previously analyzed in our laboratory utilizing the Illumina 610Quad SNP array and reported with a pathogenic abnormality. In addition to the causative $\mathrm{CNC}$, each patient had between 4 and 12 CNCs identified. All of the CNCs reported as abnormal had a score between 17 to 24 points, and 45 benign CNCs scored 11 or lower. We are expanding our analysis to additional patients which will also include CNCs reported as of unknown clinical significance.

The identification of a pathogenic or benign CNC without previously well defined clinical significance, poses a burden to clinicians, counselors and families. A clear and objective score system would not only facilitate interpretation but provide health professionals with tools for patient management. Our goal is to encourage discussion and standardization for the analysis of CNCs detected by microarray in the phenotypically abnormal patient. Any such system will have to be re-evaluated and changed accordingly once new discoveries on mechanistic disease pathways are described.

\section{7 \\ Leveraging Genotype Information from SNP Microarrays for Cytogenetic Purposes}

V. Aggarwal ${ }^{\text {a }}$, O. Nahum ${ }^{\text {a }}$, E. Carron ${ }^{\text {a }}$, D. Warburton ${ }^{\text {b }}$, V. Jobanputra ${ }^{\text {a }}$ B. Levy ${ }^{a}$

Departments of aPathology and Cell Biology and ${ }^{b}$ Genetics and Development, College of Physicians and Surgeons of Columbia University, New York, N.Y., USA

SNP oligonucleotide microarray analysis (SOMA) offers simultaneous genotyping and detection of copy number state across the human genome. While the utility of genotype information may not be apparent in the cytogenetics arena, we have leveraged the genotyping data for confirmation of gains and losses as well as for uncovering long contiguous stretches of homozygosity (LCSH) which indicate uniparental isodisomy (UPID) and consanguinity. Using the Affymetrix SNP platform, relative allele difference is calculated from polymorphic allele calls and dosage and is represented as a simple algebraic formula $\mathrm{A}-\mathrm{B}=0$. The relative allele difference is plotted on the output graph by assigning homozygous AA alleles to track 1, heterozygous AB alleles to track 0 and homozygous $B B$ alleles to track -1 . In a global view of each chromosome, all 3 tracks $(1,0$ and -1$)$ appear populated. Allele combinations will always show equal dosage and thus for deleted regions, only 2 allele tracks are visible corresponding to homozygosity with a single allele (A) at position 0.5 and a single

Preprint Cytogenet Genome Res 128/4/2010

Abstracts will be available online, free of charge 
allele (B) at position -0.5 , i.e. half the dosage. All 4 possible SNP tracks are observed in duplicated regions: $\mathrm{AAA}, \mathrm{AAB}, \mathrm{ABB}$ and $\mathrm{BBB}$ at positions $1.25,0.25,-0.25$ and -1.25 respectively. Allele dosage differentiation is a powerful secondary internal analytical tool for confirming DNA copy number changes and may, in some cases, reduce the need for additional follow-up in the proband. Furthermore, triploidy, which is usually not detected by aCGH platforms, can easily be identified by the presence of 4 SNP tracks across all autosomes. The DNA dosage component is required to differentiate deletion-based loss of heterozygosity (LOH) from copy neutral LCSH which is observed in consanguinity and UPID. Multiple LCSH regions across multiple chromosomes and $>8 \mathrm{Mb}$ are an indication of consanguinity while a single large $\mathrm{LCSH}$ region of greater than $10-15 \mathrm{Mb}$ most likely indicates UPID. Parental SOMA allows for identification of uniparental heterodisomy in the proband as well as determination of parentof-origin for deleted regions. The advantages provided by a hybrid array will be discussed and illustrated.

\section{8 \\ CNV and Regions of Homozygosity Identified by SNP Array: Difficulties in Elucidating Clinical Significance}

E. Wohler ${ }^{\text {a }}$, N. Jinawath ${ }^{c}$, A. Hamosh ${ }^{c}$, J. Hoover-Fong ${ }^{c}$, A. Elias ${ }^{c}$, D. Valle ${ }^{c}$, D. Batista ${ }^{a-c}$

aCytogenetics Laboratory, Kennedy Krieger Institute,

bDepartment of Pathology, Johns Hopkins University, 'Institute of Genetic Medicine, Johns Hopkins Medical Institutions (JHMI), Baltimore, Md., USA

SNP microarrays are currently used for molecular cytogenetic analysis to identify copy number changes, regions of homozygosity and uniparental disomy providing precise breakpoints. Such information requires in-depth analysis to establish pathogenicity of the alterations detected. We present 3 cases which required detailed investigation and comparison to the patient's clinical features to elucidate potential correlations.

Patient 1 is a 2 -year-old female with developmental delay and abdominal situs inversus. The Illumina 610-Quad SNP array identified a heterozygous deletion in chromosome $2 \mathrm{q} 13$ $(110,201,336-110,340,339)$ that included the genes MALL and NPHP1. Such heterozygous deletions are seen in normal individuals (DGV and CHOP CNV databases). NPHP1 is part of a complex group of genes each causing nephronophthisis, a ciliopathy due to homozygous single gene mutation/deletion or compound heterozygous mutations occurring in a single NPHP gene. Interestingly, changes in NPHP2 have been associated with situs inversus. While our patient has deletion of 1 allele of NPHP1, a mutation might be present on the other. Alternatively, the heterozygous deletion could be related to her phenotype by a complex gene interaction where allelic variants at multiple loci contribute to disease.

Patient 2 is a 16 -month-old male with short stature. He scored below the 3rd percentile in height and weight and had decreased levels of IGF-1 and IGFBP3. SNP array showed a single 5.5-Mb region of homozygosity on chromosome $7 \mathrm{p} 14.3 \mathrm{p} 15.1$. Within this area is the gene GHRHR, growth hormone-releasing hormone receptor, which might explain the patient's short stature.
Patient 3 is a 20-day-old female with an uncomplicated postnatal course for the first 15 days of life who subsequently developed acute encephalopathy with seizures. SNP array showed duplication of 11q22.3 $(103,544,199-104,804,934)$ which included the caspase gene family which regulate apoptosis and inflammatory processes. We are investigating if the duplication produced a gain of function that may have triggered an exaggerated inflammatory response to environmental factors such as pathogens or hypoxia resulting in the brain injury. These cases highlight the need for thorough analysis of microarray results in combination with detailed phenotypic information and appropriate confirmatory testing to investigate the potential significance of the abnormalities identified.

\section{9 \\ Subtelomeric CNV: Mechanisms of Chromosome Breakage and Repair}

K. Rudd, Y. Luo, K. Hermetz

Department of Human Genetics, Emory University School of Medicine, Atlanta, Ga., USA

Subtelomeric rearrangements were among the first copy number variations (CNVs) identified, now routinely detected with array and FISH testing. Between 3-6\% of children with previously idiopathic mental retardation are diagnosed with a pathogenic subtelomeric rearrangement. Despite the impact of subtelomeric $\mathrm{CNVs}$ on human health, the forces underlying their formation remain unknown. To uncover the mechanisms of subtelomeric breakage and repair, we performed a large-scale analysis of subtelomeric breakpoints via combined high-resolution array comparative genome hybridization (CGH), breakpoint cloning, and comprehensive sequence analysis. Using a custom oligonucleotide array, we mapped subtelomeric rearrangements in 110 patients: 49 terminal deletions, 7 interstitial deletions, 3 terminal duplications, 25 unbalanced translocations, and 26 deletions adjacent to inverted duplications, resolving breakpoints to a few hundred basepairs at each site. To isolate the repaired genomic structure and identify breakage sites, we are cloning and sequencing breakpoint junctions, with 20 junctions sequenced to date. In our analysis of translocation junctions, we find that the predominant mechanism of repair is non-homologous end joining, but we have also identified a recurrent translocation between highly identical translocating segments (95\% identical across $\sim 5 \mathrm{~kb}$ ), implicating homologous recombination in at least some rearrangements. Several terminal deletion breakpoints have microhomology to the telomere repeat (TTAGGG)n, suggesting that complementarity to the RNA component of telomerase may be involved in the synthesis of a new telomere. Inverted duplications adjacent to terminal deletions are detected via array CGH as a terminal loss and an adjacent gain. In 6 cases we cloned and sequenced the breakpoint junctions, confirming the presence of a disomic segment in between inverted duplications. We identified regions of inverted microhomology at the breakpoints, consistent with a hairpin/strand-invasion repair mechanism. Our large collection of defined subtelomeric breakpoints also allows us to identify sequence motifs that may promote genomic instability at subtelomeres; we find an enrichment of G-quadruplex DNA and tan- 
dem repeats at breakage sites. This detailed analysis of subtelomeric breakage and repair will unravel the mechanisms of chromosomal rearrangement at these and other loci that give rise to human copy number variation.

\section{0}

\section{Towards a CNV Atlas for Human Development}

C.L.Martin ${ }^{\text {a }, ~ E . B . ~ K a m i n s k y ~}{ }^{\text {a }}$, B. Bunke ${ }^{\text {a }}$, D. Kunig ${ }^{\text {a }}$, V. Kaul $^{\text {a }}$, K. Huang a , D. Saul ${ }^{\text {b }}$, D.L. Pickering ${ }^{\text {c }}$, D.M. Golden ${ }^{\text {c }}$, E. Aston ${ }^{d}$, T.J. Gliem ${ }^{\mathrm{e}}$, T. Ackley ${ }^{\mathrm{f}}$, S. Huang ${ }^{\text {g, J.C. Barber }}{ }^{\mathrm{g}}$, J.A. Crolla ${ }^{\mathrm{g}}$, R. Iyer ${ }^{\mathrm{f}}$, E.C. Thorland ${ }^{\text {, }}$, A.R. Brothman ${ }^{\text {d, W. Sanger }}{ }^{\text {, }}$, M.R. Rossi ${ }^{\text {a }}$, M.K. Rudd a , S. Aradhya b , D.H. Ledbetter ${ }^{\text {a }}$

aEmory University, Atlanta, Ga., ${ }^{\mathrm{b}}$ GeneDx, Gaithersburg, Md., 'University of Nebraska, Omaha, Nebr., ${ }^{\mathrm{d} A R U P}$ Laboratories, Salt Lake City, Utah, e'Mayo Clinic, Rochester, Minn., fUniversity

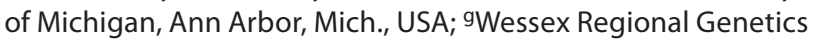
Laboratory, Salisbury, UK

Genome-wide oligonucleotide microarrays have been successfully implemented in clinical cytogenetic diagnostics. These arrays typically have a resolution of $\sim 200 \mathrm{~kb}$, resulting in a 10-100fold improvement in resolution compared to G-banding. Clinical interpretation of the chromosomal imbalances detected by arrays in individuals with abnormal phenotypes has been aided by studies of copy number variation in normal populations. We have analyzed data from 15,753 whole-genome arrays from 7 clinical diagnostic laboratories that are members of the International Standard Cytogenomic Array (ISCA) consortium. Goals of this consortium include the standardization of cytogenetic array testing and deposition of data into a public database for use by the clinical and research communities. In accordance with ISCA standards, arrays were custom designed with genome-wide backbone coverage in addition to high density coverage of known clinically relevant regions and genes. Array analysis was carried out on individuals with phenotypes including mental retardation, autism, developmental delay, dysmorphic features, and/or multiple congenital anomalies. Of the 4,614 imbalances detected within the reporting criteria, we found pathogenic abnormalities in $59.7 \%$ (17.5\% of patients) based on known clinically relevant regions, gene content, inheritance, and size. We observed many of the recently described recurrent disorders such as $16 \mathrm{p} 11$ microdeletions (63 cases) and 17q12 imbalances (18 deletions and 21 duplications). For $30 \%$ of the imbalances ( $8.8 \%$ of patients), the abnormality was classified as uncertain due to insufficient evidence. For example, duplications of $16 \mathrm{p} 11.2$ were considered uncertain since evidence is still emerging for their association with an increased risk of autism. As expected, many common, known benign copy number variants (bCNVs) were identified, including imbalances within segmental duplications, such as the DEFB gene cluster on 8p23.1. Of the 1,409 imbalances with known inheritance, 565 imbalances (40.1\%) were determined to be de novo. Of the inherited cases, many were inherited from a similarly affected parent or a parent carrying the balanced form of the rearrangement. Comparison of data such as these generated from clinical testing of individuals with abnormal phenotypes to that generated in normal populations will help to establish a gene dosage map of copy number variation for normal and abnormal human development.

\section{1 \\ Next Generation Sequencing (NGS) in Mendelian Cytogenetics}

N. Tommerup ${ }^{\text {a }, ~ S . C . ~ M i n o c h e r h o m j i ~}{ }^{\text {, }}$, L. Klitten ${ }^{\text {a }}$, L. Nazaryan ${ }^{\text {a }}$ R. Buonincontri ${ }^{\text {a }}$, R.S. Møller ${ }^{\text {a }}$, H. Hjalgrim ${ }^{\text {b }}$, K.F. Henriksen ${ }^{\text {a }}$, H. Mølgård Nielsen a , I. Bache a , A. Silahtaroglu a , C. Hansen a, M. Bak ${ }^{\mathrm{a}}$

aWilhelm Johannsen Centre for Functional Genome Research, Department of Cellular and Molecular Medicine, University of Copenhagen, Copenhagen, ${ }^{\text {b}}$ Danish Epilepsy Centre, Dianalund, Denmark

The rapid expansion of sequencing capacities has been predicted to revolutionize genomics, genetics, molecular biology and medicine. We illustrate some of the potentials of the Ilumina Genome Analyzer in Mendelian cytogenetics: We have previously mapped numerous disease-associated balanced reciprocal rearrangements (DBCRs) to BAC level by FISH. Traditionally, the next step towards the isolation of a breakpoint would be a combination of usually cumbersome strategies, e.g. Southern blotting and long range PCR, in order to detect junction fragments which could be cloned. One of the new opportunities provided by NGS is direct detection of translocation and inversion breakpoints by paired-end sequencing. Still, with the sequencing capacity and cost of today, this may have to be preceded by an enrichment step, by flow sorting of specific chromosomes, or by prior hybridization of patient DNA (300-400-bp fragments) to custom made NimbleGen oligonucleotide arrays covering breakpoint detecting BACs which will be illustrated in the presentation.

For DBCRs with no available FISH data, we have exploited mate-pair sequencing where we can enrich for and sequence fragments several kilobases in size, and thus reach a decent coverage in a single lane of the Genome Analyzer flow cell. We also illustrate some of the potential pitfalls - the presence of repetitive sequences at the breakpoints and existing gaps in the annotated genome - which could make NGS cloning of breakpoints difficult in specific cases.

A huge advantage of NGS is the broad potential for functional analyses, including global analyses of the transcriptome by RNASeq, of DNA methylation by MeDIP-Seq and of histone modifications and transcription factor binding sites by ChIP-Seq. Thus, with the same technical platform, we can both map single breakpoints down to sequence level, and analyze the transcriptional status and epigenetic marks at these breakpoints.

\section{2 \\ Human Genome Structural Variation: From Cytogenetics to Sequence}

\section{E.E. Eichler}

Department of Genome Sciences and Howard Hughes Medical Institute, University of Washington, Seattle, Wash., USA

Cytogenetics has long appreciated that genomic structural variation is an important aspect in our understanding of the molecular basis of common disease. The human genome is particu-

Preprint Cytogenet Genome Res 128/4/2010

Abstracts will be available online, free of charge 
larly enriched in complex patterns of structural variation (inversions, deletions and duplications), although accurately and systematically characterizing such variation in both disease and normal human genomes remains an unmet challenge. Using cytogenetic, microarray and sequencing approaches, I will summarize our current understanding of the pattern of normal genome structural variation and contrast it with what we have observed in children with intellectual disability and developmental delay. Our analyses have identified several rare microdeletions and microduplications significantly associated with various pediatric phenotypes (e.g. 17q12, 17q21.31, 15q13.3, 15q24 and 1q21.1). We show that the breakpoints of these rearrangements lie in flanking segmental duplications that are frequently structurally polymorphic in the human population. Detailed characterization of patient phenotypes, as well as comparisons with other studies, reveals extreme variability in expressivity suggesting that each molecular lesion may manifest in a variety of pediatric and adult phenotypes, including autism, schizophrenia, mental retardation, diabetes and/or epilepsy. An analysis of $>4,000$ 'normal' individuals reveals that this form of large-scale duplication-mediated structural variation is individually rare but collectively common over many sites. Using next-generation sequencing methods, I will discuss novel approaches to systematically characterize such variation at the whole-genome level and emphasize the need for orthogonal and complementary approaches to systematically characterize this variation in the future.

\section{3 \\ Mosaic Trisomy 13: Understanding the Origin Using SNP Array}

N. Jinawath a , R. Zambrano a , E. Wohler ${ }^{\text {b }}$, D. Batista ${ }^{\text {a-c }}$

anstitute of Genetic Medicine, Johns Hopkins Medical Institutions (JHMI), 'bennedy-Krieger Institute, 'Department of Pathology, JHMI, Baltimore, Md., USA

Trisomy 13 occurs in 1/10,000 live births and mosaicism accounts for $5 \%$ of these cases. Phenotype and outcome of mosaic trisomy 13 are variable and poorly understood. We present 2 cases of mosaic trisomy 13 with similar facial features, failure to thrive and cardiac anomalies. Patient 1 had karyotype $47, \mathrm{XX},+13[10] / 47, \mathrm{XX},+\operatorname{mar}[40]$. The marker identified by metaphase FISH with CEP13/21 and WCP13 contained only the centromeric material of chromosome 13. The karyotype of patient 2 was $47, \mathrm{XX},+13[1] / 46, \mathrm{XX}[49]$. FISH with LSI13 demonstrated $24 \%$ trisomic interphases. To elucidate the origin and underlying pattern of inheritance, we analyzed the patients and their parents using Illumina SNP array. Copy number represented by log R ratio showed a subtle increase between 2 and 3 copies in both patients. The genotypes were then analyzed with software packages SNPtrio (inheritance patterns in family trios) and SNPduo (identity by state between 2 individuals). Patient 1 had maternal heterodisomy from centromere to genomic location $83.40 \mathrm{Mb}$, maternal isodisomy from $83.40 \mathrm{Mb}$ to $105.63 \mathrm{Mb}$ (13q31.1q33.2), and maternal heterodisomy from $105.63 \mathrm{Mb}$ to telomere. These findings in combination with the B-allele frequency showing 3 different haplotypes along chromosome 13 with the exception of the region between $83.40-105.63 \mathrm{Mb}$ that had only 2 different haplo- types, suggested origin of the trisomy 13 in maternal meiosis I with crossovers at $83.4 \mathrm{Mb}$ and $105.63 \mathrm{Mb}$. In patient 2, two copies of chromosome 13 were shared identically between the patient and mother, suggesting maternal heterodisomy. B-allele frequency demonstrated 3 haplotypes across the entire chromosome 13. This trisomy also originated in meiosis I but without crossover, which may have predisposed to non-disjunction. The mechanism underlying UPD in both patients appeared to be trisomic rescue. Additionally, based on the algorithm derived by Conlin et al., we calculated the trisomic percentage as $40 \%$ and $30 \%$ respectively, which should represent the direct measurement of mosaicism without culture artifact. In summary, SNP array provides both genotypes and copy number information and is useful in studying mechanisms of complex inheritance underlying mosaic aneuploidy and UPD, which if done collectively may lead to a better genotype-phenotype correlation of mosaic cases.

\section{4 \\ Increasing the Yield and Accuracy of Karyotypes from Spontaneous Abortion Specimens}

\author{
V. Jobanputra ${ }^{a}$, C. Esteves ${ }^{\text {a }}$, A. Sobrino ${ }^{\text {b }}$, S. Brown ${ }^{\text {, }}$, J. Kline a, \\ D. Warburton ${ }^{\text {a }}$
}

${ }^{a}$ Columbia University Medical Center, ${ }^{b} \mathrm{New}$ York Presbyterian Hospital, New York, N.Y., CUniversity of Vermont, Burlington, Vt., USA

Cytogenetic analysis of a spontaneously aborted conceptus provides valuable clinical information for couples who experience recurrent spontaneous abortions. Standard cytogenetic analysis of spontaneous abortions requires culture and karyotype of chorionic villi or fetal tissues. Two major problems associated with culture of fetal tissues are failure to grow in culture and possibility of maternal cell contamination. In a previous study [Jobanputra et al., 2002] we have validated a multiplex interphase FISH probe set for chromosomes $13,15,16,18,21,22, \mathrm{X}$ and $\mathrm{Y}$ as a screen for common aneuploidies in uncultured tissues from spontaneous abortions and have shown that this probe set will detect $80 \%$ of chromosome abnormalities in spontaneous abortions.

We have now used this approach in 2 research studies involving karyotyped spontaneous abortions as well as samples received in our clinical lab. In total we analyzed uncultured tissues from 162 samples showing a 46,XX karyotype and from 230 samples which failed to grow or were microbially contaminated. From specimens karyotyped as 46,XX, FISH showed a normal female in 117, a normal male in 13 and an abnormal complement in 32. Thus $27.8 \%$ of 46 ,XX karyotypes were demonstrably incorrect, and $19.8 \%$ of these were abnormal. In specimens with culture failure, FISH showed a normal female in 71, normal male in 77 and an abnormal complement in 82 (35.7\%). The abnormalities in both groups were distributed as expected, except for an unusually high number with monosomy 21 or 15 (8 or $2 \%$ of all specimens). This suggests that monosomies may often fail to grow in culture.

Maternal cell contamination studies were performed in 70 of the cultures karyotyped as normal female with maternal DNA available. Twenty-nine cultures were maternal, 33 were fetal and 8 were a maternal/fetal mixture. 
These results demonstrate the unreliability of a 46,XX result in cultures from spontaneous abortion specimens, even when specimens are carefully dissected to find fetal material. FISH showed an abnormal result in $\sim 20 \%$, even though the probe set does not detect all abnormalities. The results also demonstrate the utility of using FISH on saved suspensions from cultures that fail to yield a karyotype: $\sim 36 \%$ could be classified as abnormal.

\section{5 \\ Variable Mechanisms of Chimera Formation Identified by SNP Array Analysis}

L.K. Conlin a , B.D. Thiel a , S. Mulchandani ${ }^{\text {a }, ~ D . N . ~ H a r t u n g ~}{ }^{\text {b }}$, L. Medne ', E. Zackai c, C.G. Bonnemann ', B.K. Burton b, M.A. Deardorff ${ }^{c}, H$. Hakonarson ${ }^{c}$, N.B. Spinner ${ }^{\text {a }}$

aDepartment of Pathology and Laboratory Medicine, 'Department of Pediatrics, The Children's Hospital of Philadelphia, Philadelphia, Pa., 'b Department of Genetics, Birth Defects, and Metabolism, Children's Memorial Hospital, Chicago, III., USA

Chimerism, the presence of genetically distinct cell lines derived from separate zygotes, is believed to be rare. The detection of chimerism is difficult using cytogenetic analysis, especially in cases that involve identical chromosomal constitution between the 2 cell lines. Identification of chimerism often requires molecular analysis, and the use of genome-wide SNP arrays makes the differentiation of chimerism possible. We have studied over 3,000 patients in our clinical CytoGenomics laboratory using a SNP array platform and identified 4 individuals with chimerism. The presence of a cell line with complete uniparental isodisomy was seen in 2 patients, with a maternal origin in 1 individual and paternal in the other. These 2 individuals had clinical features that were consistent with an imprinting disorder. The first patient, an XX/XY phenotypic male with a Prader-Willi-like phenotype had complete maternal uniparental isodisomy, suggesting parthenogenetic chimerism. The second patient presented with Beckwith-Wiedemann syndrome, and had a 46,XX karyotype. Parental genotyping confirmed complete paternal uniparental isodisomy in 1 cell line, consistent with a mechanism of androgenetic chimerism. The remaining 2 cases presented as monochorionic, sex-discordant twins. SNP array results revealed a pattern of genotypes in both patients that were consistent with fusion of a 46,XX and a 46,XY embryo, with subsequent fission and formation of twins. The identification of 4 chimeric individuals $(0.1 \%)$ in our cohort was surprising, given the rarity of this finding. While chimerism is often suspected because there are both $46, \mathrm{XX}$ and 46,XY cell lines present in 1 individual (as in the monochorionic twins), the use of SNP arrays provides the ability to diagnose unexpected instances of chimerism, and future studies may reveal more about these unusual findings.

\section{6 \\ SNP Microarray Analysis Offers New Insights into the Accuracy of FISH Diagnostics in Preimplantation Embryos}

B. Levy ${ }^{\mathrm{a}, \mathrm{b}}$, L.E. Northrop ${ }^{\mathrm{b}, \mathrm{c}}$, N.R. Treff ${ }^{\mathrm{b}, \mathrm{c}}$, R.T. Scott Jr. ${ }^{\mathrm{b}, \mathrm{c}}$

aDepartment of Pathology and Cell Biology, College of Physicians and Surgeons of Columbia University, New York, N.Y. ${ }^{b}$ Reproductive Medicine Associates of New Jersey, Morristown, N.J., 'Division of Reproductive Endocrinology and Infertility, Department of Obstetrics Gynecology and Reproductive Science, University of Medicine and Dentistry of New Jersey, Robert Wood Johnson Medical School, New Brunswick, N.J., USA

FISH was among the first and has been the most widely used technology to screen for aneuploidy in human embryos. Although selection of chromosomally normal embryos has the potential to improve outcomes for patients undergoing IVF, the clinical impact of aneuploidy screening by FISH has been controversial. There are many possible reasons why FISH has failed to show meaningful improvements in clinical outcome including (i) mosaicism, (ii) embryo self correction, (iii) preferential segregation of aneuploid chromosomes to the trophectoderm and (iv) fundamental limitations intrinsic to single cell FISH diagnostics. To date, there has not been direct assessment of the negative predictive value of FISH-based aneuploidy screening for an embryo's reproductive potential. Instead, studies have re-evaluated ongoing blastocysts that were diagnosed as abnormal by FISH at the cleavage stage and subsequently observed normalcy rates ranging from $10 \%$ to $71 \%$. Discrepant results have been attributed to mosaicism and self correction despite no experimental evidence to support these explanations. The present study used a SNP-based microarray with 262,000 probes to re-evaluate morphologically normal blastocysts that were diagnosed as aneuploid by FISH at the cleavage stage. Multiple sections of the blastocyst (inner cell mass +3 separate sections from the trophectoderm) were analyzed to determine the contribution of mosaicism and preferential segregation of aneuploid cells to the trophectoderm. The SNP genotype information provided the first opportunity to evaluate self-correction mechanisms involving extrusion (trisomy rescue) or duplication (monosomy rescue) of aneuploid chromosomes resulting in uniparental isodisomy (UPID). Of all blastocysts evaluated $(n=50), 58 \%$ were euploid in all sections despite an initial aneuploid FISH result. Aneuploid blastocysts displayed no evidence of preferential segregation of abnormalities to the trophectoderm. In addition, extrusion or duplication of aneuploid chromosomes resulting in UPID did not occur. These findings support the conclusion that cleavage stage FISH technology is poorly predictive of aneuploidy in morphologically normal blastocysts. It is therefore likely that FISH on cleavage stage embryos results in the erroneous disposal of reproductively competent blastocysts. At minimum, retesting by an alternate technique of morphologically normal blastocysts that develop despite an aneuploid cleavage stage FISH diagnosis is strongly recommended.

Preprint Cytogenet Genome Res 128/4/2010

Abstracts will be available online, free of charge 


\section{7}

New ACMG Laboratory Guideline for Turner Syndrome: Positive Impact on Patient Care at MUSC

D.J. Wolff

Medical University of South Carolina, Charleston, S.C., USA

Recently, the American College of Medical Genetics (ACMG) Laboratory Quality Assurance committee published a diseasespecific guideline that provides laboratory guidance for the diagnosis/study of patients with Turner syndrome and its variants. At MUSC, implementation of the ACMG recommendation for postnatal studies involved the initiation of 2 new procedures in our laboratory: (1) reflexive X/Y FISH testing following a karyotype finding of 45,X, and (2) reflexive XIST FISH testing when a marker chromosome is determined to be derived from an X chromosome. To establish the clinical impact of these policy changes, the appropriate FISH testing was retrospectively reviewed/performed on all 2009 cases with a 45,X karyotype or a marker X chromosome. Seven cases were identified with a 45,X karyotype; one of which showed Y chromosome signal in $4 \%$ of cells. Assessment of 115 metaphase cells documented a small marker chromosome in 2 cells. Pelvic ultrasound of this 16-year-old patient demonstrated a small uterus and no visualized ovaries. Surgery is scheduled to remove the dysgenetic gonads. X/Y FISH studies on the remaining 6 patients revealed results consistent with only $45, \mathrm{X}$ cells with no evidence for mosaicism. There were 2 structurally abnormal X cases from 2009 for which FISH for XIST was considered. One had a $45, \mathrm{X} / 46, \mathrm{X}, \mathrm{r}(\mathrm{X})$ karyotype with ring breakpoints designated as p22.3 and q24 and, given the banding pattern, XIST FISH was deemed unnecessary. The second case was a newborn patient being evaluated for hypoplastic left heart syndrome with a $45, \mathrm{X} / 46, \mathrm{X}, \operatorname{del}(\mathrm{X})(\mathrm{q}$ ?21) karyotype. FISH with a probe for the XIST gene documented that XIST was present on the structurally aberrant X chromosome. In conclusion, institution of the ACMG Laboratory Quality Assurance committee's recommendations for laboratory study of Turner syndrome has had a positive impact on patient care.

\section{8}

Molecular Characterization of Chromosome Aberrations in 146 Patients with Phelan-McDermid Syndrome by Array-CGH

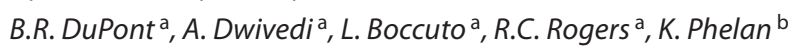

${ }^{\mathrm{a} G r e e n w o o d ~ G e n e t i c ~ C e n t e r, ~ G r e e n w o o d, ~ S . C ., ~}{ }^{\mathrm{b}}$ Molecular

Pathology Laboratory Network, Maryville, Tenn., USA

Phelan-McDermid syndrome (PMS) or 22q13 deletion syndrome is a microdeletion syndrome characterized by facial dysmorphism, moderate to profound developmental delay, hypotonia, seizures, autistic-like behavior and delayed or absent speech. The loss of the distal long arm of chromosome 22 can arise from a simple deletion, an unbalanced translocation or from ring chromosome formation. It occurs with equal frequency in males and females and has been reported in both mosaic and non-mosaic forms. The conventional methods of detection of this genetic ab- erration are high resolution chromosome analysis and fluorescence in situ hybridization (FISH). However, the deletions in some cases have been missed by these methodologies and were detected later by microarray analysis. With the help of the $22 \mathrm{q} 13$ Deletion Foundation and others, we have collected blood samples and clinical information on 146 probands, available parents and family members. We report here a custom chromosome 22 oligo array-CGH analysis (OGT, UK) of 146 deletion $22 \mathrm{q} 13$ probands to determine the size of the deletions in patients with PMS. The custom array spans 319 genes (of which 252 are protein coding genes) between the regions $35-50 \mathrm{Mb}$ on chromosome 22. Of the 146 probands analyzed, 105 had simple deletions, the largest missing 9.22 $\mathrm{Mb}$ (between breakpoints $40.475 \mathrm{Mb}$ and $49.69 \mathrm{Mb}$ ); the smallest missing $0.13 \mathrm{Mb}$ (between breakpoints $49.27 \mathrm{Mb}$ and $49.69 \mathrm{Mb}$ ). Three were found to have interstitial deletions and 2 were found to have duplications. Nine cases were found to have a duplication (ranging from $0.2-6.8 \mathrm{Mb}$ ) proximal to the deletion (ranging from 0.4-7.2 Mb) on chromosome 22. The known genes which are deleted in the majority of cases are FBLN1, ATXN10, GTSE1, TRMU, BRD1, ALG12, CRELD2, MLC1, CHKB, ARSA, $S H A N K 3$ and $A C R$. We also report here parent-of-origin analysis for those probands from which we have parental samples. Only $40 \%$ of our initial studies using variants within SHANK3 were informative with $67 \%$ paternal deletions and $33 \%$ maternal deletions. We currently are conducting further analysis on the $60 \%$ of cases which were uninformative. Genotype/phenotype analyses are underway to collect information that will be used to highlight candidate genes associated with clinical, neurological and other features.

\section{Poster Presentations Constitutional Cytogenetics}

\begin{abstract}
39
Array Comparative Genomic Hybridization Identifies New Candidate Genes for Idiopathic Mental Retardation

A. Bewell ${ }^{\text {a }}$, C. Jackson ${ }^{\text {b }, ~ G . ~ R i c e ~}{ }^{c}$, G. Raca ${ }^{\text {b, d }}$, J. Laffin ${ }^{\text {b, c }}$

aUniversity of Wisconsin-Madison Genetic Counseling Program, buW Cytogenetics Services, Wisconsin State Laboratory of Hygiene, 'Department of Pediatrics, University of WisconsinMadison, ${ }^{\mathrm{d} D e p a r t m e n t}$ of Pathology and Laboratory Medicine, University of Wisconsin-Madison, Madison, Wisc., USA
\end{abstract}

Approximately $2-3 \%$ of the population is affected by intellectual and developmental disabilities (IDD) with the vast majority of this subpopulation having idiopathic mental retardation. There have been hundreds of genes implicated in mental retardation most accounting for only a fraction of a percent. Even with an abundance of potential genetic possibilities for $80 \%$ of individuals with non-syndromic mental retardation the etiology remains unknown. 
We present here data from an ongoing project to identify candidate genes for idiopathic intellectual and developmental disabilities in individuals who have apparently normal chromosome and Fragile X molecular testing results. The methods use a combination of X-inactivation (define a likelihood of X-linked disease), high density oligonucleotide array comparative genomic hybridization (aCGH) (identify genomic copy number variants) and sequencing (survey of common X-linked genes). The current results have identified 5 candidate genes, AUTS2, ZNF277, SNTG2, CSMD1, and SRGAP1, in 3 probands.

The first proband is a 5-year-old female with hypotonia, global developmental delay, and microcephaly. Three deletions were identified by aCGH that involve the SNTG2 gene $(13.7 \mathrm{~kb})$ on chromosome 2, CSMD1 (8 kb) on chromosome 8, and SRGAP1 (13 $\mathrm{kb})$ on chromosome 12 .

The second proband is a 16-month-old male with hypotonia, global developmental delay, and growth failure. A single 19.4-kb deletion involving ZNF277 on chromosome 7 was identified by aCGH.

The third proband is an 11-month-old male with growth retardation and global developmental delay. The family history is significant for 2 brothers with global developmental delay, a brother with attention deficit hyperactivity disorder, and both parents with learning disabilities. aCGH identified a 33.7-kb deletion involving the AUTS2 gene on chromosome 7.

Future studies will be necessary to determine the impact of these deletions on development and the IDD phenotype.

\section{0}

\section{Cytogenetics of Mental Retardation}

D. Chandel, V.R. Hydrabadi, M.V. Rao

Human Genetics Unit, Department of Zoology, Gujarat

University, Ahmedabad, Gujarat, India

It is well known that chromosome imbalance, especially of autosomes can cause multiple congenital anomalies and mental retardation. Some of these phenotypes are easily recognized on clinical grounds (such as Down syndrome), while others are not so easily recognized, either due to rarity or a wide spectrum of abnormalities. The etiological factors involved in the remaining vast majority of such cases are unknown. Cytogenetic studies in mentally retarded individuals lead to their better management and accurate genetic counseling. This study was therefore undertaken to determine the chromosome anomaly, if any, as a cause of mental retardation in the patients referred from regions of Western India.

In the present study 222 mentally retarded cases were investigated, and were first examined by a consultant pediatrician. Clinical features, family history and detailed pedigrees were recorded in a proforma. $116(52.3 \%)$ cases were confirmed with Down syndrome, which made the greatest contribution to mental handicap. Free trisomy accounted for 112 (96.5\%) cases, translocation for 4 (3.5\%) Down syndrome cases. A significantly higher number of males were observed in Down syndrome cases. Six (2.7\%) cases were observed to have normal chromosome variants. The most common variant was inv(Y). Four unique cases were observed in the present study:
Case 1: 46,XX,t(21;21),inv9qh

Case $2: 47, X Y,+21, t(11 ; 21)(11 p t e r \rightarrow 11 q 13.1:: 21 p 12 \rightarrow 21$ pter;21q ter $\rightarrow 21 \mathrm{p} 12:: 11 \mathrm{q} 13.1 \rightarrow 11 \mathrm{qter})$

Case 3: 46,XX,dup(16)(?q13 $\rightarrow$ ?qter)

Case 4: 46,XX,t(15;18)(p13;q21.3)

The observations and their implications will be discussed.

\section{1}

Prenatal Diagnosis of de novo Partial Trisomy 2q

A.C. Kuskucu ${ }^{\text {a }}$, K. Yararbas ${ }^{\text {a }}$, G. Yildirim ${ }^{\text {b }}$, O. Sahin ${ }^{\text {b }}$, A. Gul ${ }^{\text {b }}$, Y. Ceylan ${ }^{b}$

aBakırköy Women and Children's Health Education and Research Hospital Dept. Medical Genetics, and bBakırköy Women and Children's Health Education and Research Hospital Dept. Gynecology and Obstetrics Division of Perinatology, İstanbul, Turkey

Partial trisomy of chromosome $2 \mathrm{q}$ is a rare chromosomal aberration with only 2 prenatally diagnosed cases published. Phenotype is variable in previously reported cases. Here we would like to report a fetus with partial trisomy $2 \mathrm{q}$ detected by amniocentesis at 16 weeks gestation with cystic hygroma and ventricular septal defect. The pregnancy terminated and additional autopsy findings were cleft palate, ambiguous genitalia and syndactyly between 4th and 5th toe. Chromosome analysis of fetal blood sample and fluorescent in situ hybridization studies were performed to confirm that the extra chromosomal material originated from chromosome 2 itself.

\section{2 \\ Origin and Phenotypic Consequences of Mosaic 45,X/47,X,dup(Y)(q11.2q12),+i(18)(p10) Karyotype}

S. Moore, R. Cooper, H. Lawce, S. Olson

Clinical Cytogenetics Laboratory, Oregon Health and Science University, Portland, Oreg., USA

Turner Syndrome has an incidence of approximately 1/2500 and affected girls often present neonatally with heart and kidney abnormalities and neck webbing. Tetrasomy $18 \mathrm{p}$ is a rare condition $(1 / 140,000)$ that may present with multiple congenital abnormalities, including hypospadias and cryptorchidism. Both of these chromosomal abnormalities may exist in mosaic form with normal cells. We received a peripheral blood specimen on a 4-year-old male with webbed neck, hypospadias, cryptorchidism and inguinal hernia. Banding at the 700-850 band level of resolution identified 2 abnormal clones in 20 metaphase cells, with no normal cells found. One clone had a 45,X karyotype. A second clone had a duplication of the distal region of the $\mathrm{Y}$ chromosome (satellite III region) and an extra abnormal chromosome, isochromosome 18p, i.e. $\operatorname{mos} 45, \mathrm{X}[16] / 47, \mathrm{X}, \operatorname{dup}(\mathrm{Y})(\mathrm{q} 11.2 \mathrm{q} 12),+\mathrm{i}(18)$ (p10)[4]. FISH was consistent with the metaphase results. Synthesis of the metaphase and FISH results indicates that both clones comprise roughly $50 \%$ of the cell population, suggesting either an early event or a growth advantage in one clone in the blood. DAPI

Preprint Cytogenet Genome Res 128/4/2010

Abstracts will be available online, free of charge 
marker regions were identical, within resolution, between the 2 clones, supporting a common origin. The phenotype, with characteristics of both Turner syndrome and tetrasomy 18p, supports the hypothesis of balanced mosaicism in most tissues, that is, an early event. Parental blood has been requested and the results and further studies will be discussed in the context of origin of the abnormal clones and reproductive implications for this family.

\section{3 \\ SNP-Based Genomic Microarray in the Workup of Mental Retardation}

J. Kogan, S. Zimmerman, S. Mikulich, T. Smolarek

Cincinnati Children's Hospital Medical Center, Cincinnati, Ohio, USA

Genomic microarray analysis has proven to be indispensible in the etiologic evaluation of children with mental retardation. Single nucleotide polymorphism (SNP) based genomic microarray analysis provides a very high-resolution unbiased chromosome copy number analysis in a relatively short amount of time. In addition, such microarrays provide genotype data, allowing for identification of regions of extended homozygosity, and the potential for determination of parental origin of a chromosome abnormality. In this study, DNA was analyzed from 21 children with mental retardation and their phenotypically normal parents. All children had an apparently normal karyotype by standard chromosome analysis. Among the 21 children, 3 (14.3\%) were found to have copy number abnormalities that were likely pathogenic. One patient was found to have a cryptic unbalanced subtelomere rearrangement, 1 was identified with a known microdeletion syndrome, and 1 was found to have a previously unreported microdeletion. SNP-based genomic microarray is extremely valuable in the identification of pathogenic copy number abnormalities as they apply to the etiology of mental retardation.

\section{4 \\ A Case of Bardet-Biedl Syndrome Associated with Homozygosity and Identified by Chromosome Microarray Analysis}

J.E. Wiley ${ }^{\text {a }}$, H. Kearney ${ }^{\text {b }}$, B. Ozturk ${ }^{c}$, A. Meyer ${ }^{\text {a }}$

aThe Brody School of Medicine, East Carolina University, Greenville, N.C., ${ }^{b}$ Fullerton Genetics Center, Mission Hospitals, Asheville, N.C., 'Stony Brook University, Stony Brook, N.Y., USA

Bardet-Biedl syndrome (BBS) is an autosomal recessive disease exhibiting locus heterogeneity. At least 12 genes are associated with BBS and sometimes there is more than 1 mutated gene involved. The present case is a 12-year-old male with morbid obesity, polydactyly and retinitis pigmentosa. Conventional cytogenetic studies revealed a 46,XY, cytogenetically normal male karyotype. Fluorescent in situ hybridization with DNA probes specific to the Prader-Willi/Angelman loci did not identify any deletions. However, chromosome microarray analysis (CMA) re- vealed an approximately 13.4-Mb area of homozygosity between bands q26 and q28.1 on chromosome 4. This area contains 80 known genes, including the Bardet-Biedl 7 (BBS7) and BardetBeidl 12 (BBS12) genes. Although homozygosity by descent has been seen in some cases of BBS, this is, to our knowledge, the first report involving the $B B S 7$ and $B B S 12$ genes. Homozygosity by descent should be considered when evaluating patients suspected of having autosomal recessive conditions.

\section{5 \\ Uncovering Single Gene Disorders with aCGH: An Example of a Family with Branchiootorenal Spectrum Disorder \\ M.A.Manning ${ }^{\text {a }}$, J. Kaplan ${ }^{\text {a }}$, J. Bernstein ${ }^{\text {a }}$, K.M. Chen ${ }^{\text {b }}$, A.M. Cherry ${ }^{\text {a }}$ \\ aStanford University School of Medicine, Stanford, Calif., \\ bLucile Salter Packard Children's Hospital, Palo Alto, Calif., USA}

It is well known that microarray comparative genomic hybridization (aCGH) is a precise and accurate method of screening for submicroscopic, genome-wide copy number alterations. It has become standard of care when evaluating patients with developmental delay (DD)/mental retardation (MR), dysmorphic features and multiple congenital anomalies. Effectiveness as a diagnostic tool in the evaluation of non-syndromic DD/MR, autism and growth abnormalities has also been demonstrated. Identifying DNA copy number gains or losses that predispose a patient to tumor formation or cancer has more recently been described. Another benefit of aCGH is that of uncovering single gene disorders and phenotype clarification. This latter application is highlighted by the case herein presented.

Our medical genetics service was consulted on a newborn male with multiple anomalies and family history of 'fascioscapulohumeral muscular dystrophy.' The baby was noted to have $\mathrm{L}$ microtia with absent external canal and 6 preauricular pits/tags, $\mathrm{R}$ preauricular pit, micrognathia, bilateral branchial pits, pectus excavatum and vocal cord paralysis. These findings were concerning for branchio-oto-renal (BOR) syndrome, an autosomal dominant condition caused by mutations in the EYA1 gene located on $8 \mathrm{q}$ and characterized by malformations of the ear, branchial fistulae/cysts and renal malformations. Clarification of the family history revealed that, in addition to sloping shoulders, the patient's mother and other family members with the same skeletal finding had hearing loss and ear pits. EYA1 mutation analysis and CGH were recommended. EYA1 testing was negative, however an Agilent $44 \mathrm{~K}$ oligonucleotide array detected a $2.4-\mathrm{Mb}$ deletion on chromosome 8 at $8 \mathrm{q} 13.2$ to $8 \mathrm{q} 13.3$ [arr $8 \mathrm{q} 13.2 \mathrm{q} 13.3$ $(70,268,687-72,630,611) \times 1]$. Interestingly, oto-facial-cervical (OFC) syndrome, another autosomal dominant condition, has been shown to be allelic with BOR and is characterized by hearing loss, branchial fistulae, ear pits, hypoplasia of the cervical musculature (sloping shoulders), variable MR and short stature. Thus, although incompletely evaluated at this point, this family is best classified at the OFC end of the BOR spectrum of disorders.

This case further illustrates the effectiveness of aCGH at precisely defining copy number changes and pinpointing genes crucial for syndrome characterization which can have direct implications for patient management. 


\section{6}

\section{Hybridization of a Centromere Probe to a Non-Specific Chromosome in Interphase FISH}

Y.-P. Essig ${ }^{a}$, B. Dickey ${ }^{a}$, M. Prentice ${ }^{\mathrm{b}}$, S.A. Berend ${ }^{\mathrm{a}}$

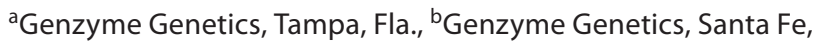

N.Mex., USA

Interphase fluorescence in situ hybridization (FISH) analysis for aneuploidy screening of prenatal cases provides a rapid result for aneuploidies involving chromosomes 13, 18, 21, X, and Y. In the majority of cases, the rapid FISH analysis gives accurate results that correlate with the subsequent cytogenetic studies; however, a small subset of cases may show discordant results where the centromere probe for $18, \mathrm{X}$, or Y hybridizes to the centromere of another chromosome. We report on an additional prenatal case in which the results of the interphase FISH analysis differ from the cytogenetic findings due to hybridization of a centromere probe to another chromosome. This case was a chorionic villus sample referred for prenatal testing due to advanced maternal age. Interphase FISH testing on a direct preparation of the villi showed results consistent with trisomy $\mathrm{X}$. The cytogenetic analysis showed a normal female chromosome complement. FISH on metaphase cells revealed that the X chromosome-specific centromere probe was hybridizing to the centromere of one chromosome 19. In 2006, we reported a case that showed similar findings. The interphase FISH results were consistent with trisomy 18 followed by a normal chromosome analysis. The FISH on metaphase cells revealed that the chromosome 18-specific centromere probe was hybridizing to the centromere of one chromosome 2 . These exceptional cases further highlight the complementary nature of the information obtained from interphase FISH and traditional cytogenetic analysis.

\section{7 \\ Implementation of a Global Platform-Independent CNV Repository}

C. Parman, S. Verma, Z. Che, R. Keshavan, S. Shams

BioDiscovery Inc., El Segundo, Calif., USA

The rapid improvement in technologies used to detect copy number variations (CNV) has led to an explosion in the amount of data being generated. Given the number of samples that have been processed at different laboratories using different platforms, collectively these datasets can be in excess of several terabytes in size. It is recognized by the scientific community that the ability to pool together results from all these studies regardless of the lab or platform has an immense value. However, effectively sharing, synchronizing, and mining these large datasets between sites can be a very challenging task. To address this issue we have introduced a 'cloud' based system to store and mine CNV data with effectively limitless storage and computational power. This system can be used to store both probe level data along with analysis results in a platform-independent manner allowing for data mining across diverse datasets to develop a better understanding of these events and their relationship to various phenotypes and subpopulations.
The cloud-based system described here runs in conjunction with a desktop application software and provides a comprehensive set of efficient queries to identify regions, genes, and functions across all samples in the database. This system currently ties together over 130 sites across the globe involved in CNV research allowing secure storage of many terabytes of data. This unique system allows all these sites to query and mine data from hundreds of thousands of samples regardless of array platform used. We will describe the architectural details and performance of the design and demonstrate the utility of this system through several examples.

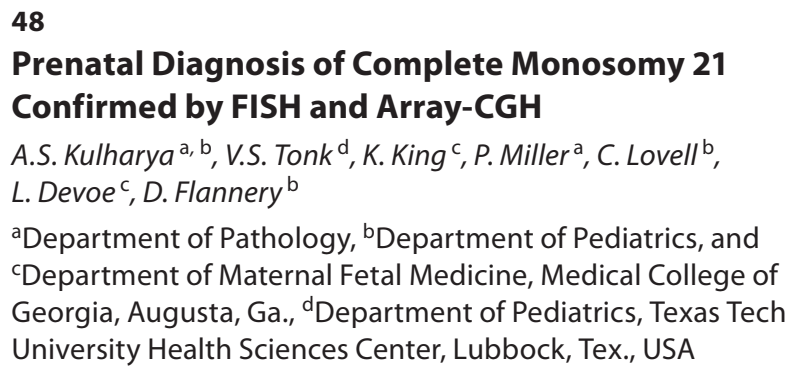

${ }^{\mathrm{a} D e p a r t m e n t}$ of Pathology, ${ }^{\mathrm{b}}$ Department of Pediatrics, and 'Department of Maternal Fetal Medicine, Medical College of Georgia, Augusta, Ga., dDepartment of Pediatrics, Texas Tech University Health Sciences Center, Lubbock, Tex., USA

Complete monosomy 21 is an extremely rare abnormality which is incompatible with life. All previously reported cases with presumed complete monosomy 21, prior to advent of FISH and array-CGH, have been ultimately partial monosomies for $21 \mathrm{q}$ with segmental aneuploidy for another chromosome. Patients with partial deletions of $21 \mathrm{q}$ present with multiple anomalies including dysmorphic features, arthrogryposis and mental retardation. The severity of effects in these cases varies with the location of the breakpoint.

We present the first documented case of complete non-mosaic monosomy 21 in a liveborn. The chromosome abnormality was diagnosed prenatally and confirmed by FISH and array-CGH. We will also present post-natal phenotypic findings in this patient.

The mother was an 18 -year-old gravida 1 , para 0 . Her medical history was significant for gastric adenocarinoma at 14 years of age which was treated with chemotherapy and gastrectomy. The history was negative for recurrent miscarriage, stillbirths, and birth defects. An ultrasound (US) at 19 weeks of gestation detected cardiac and renal anomalies, and rocker bottom feet. Cytogenetic analysis of amniotic fluid showed complete monosomy 21 with the karyotype being $45, \mathrm{XY},-21$. FISH analysis in uncultured amniocytes was also consistent with loss of chromosome 21. These findings were confirmed by 44,000 oligonucleotide arrayCGH. A high resolution maternal karyotype was normal.

Serial ultrasounds during the entire gestation period continued to show increasing number of fetal anomalies. An US performed at 33 weeks of gestation showed lemon shaped head with asymmetric growth restriction. The head circumference, biparietal diameter, abdominal circumference, femur length, and estimated fetal weight measurements were all below the 5 th percentile. The fetus was in breech presentation at this gestation and remained in this position until delivery at term via C-section.

On physical examination, the infant displayed dysmorphic features, microcephaly with ovoid-shaped skull, skeletal anomalies, arthrogryposis, and bilateral rocker-bottom feet. A third nip- 
ple and epithelialized abdominal wall defect to the left of the umbilicus was also visible. The patient died at 4 days of age.

Postnatally, the karyotype was confirmed in peripheral blood by FISH and chromosome analysis and in buccal cells by FISH analysis.

\section{9}

\section{Uncovering Hidden Cytogenetic Aberrations by Integration of Karyotyping, FISH and Chromosomal Microarray}

J. Liu a , B.A. Kozel ${ }^{\text {b }}$, M. Simon a , J.Y. Bauer ${ }^{\text {a }}$, S. Kulkarni ${ }^{\text {a }}$

${ }^{a}$ CytoGenomics and Molecular Pathology, Division of Pathology and Laboratory Medicine, Department of Pathology and Immunology, and 'bSt. Louis Children's Hospital, Department of Pediatrics, Washington University School of Medicine in St. Louis, Mo., USA

Clinical cytogenetics is the study of chromosomes, their structure and inheritance. The traditional metaphase karyotyping allows the detection of numerical and structural aberrations at a resolution of 5-10 Mb. Fluorescence in situ hybridization (FISH) is able to evaluate alterations at known chromosomal loci on a cell by cell basis. The recent prevailing microarray-based genomic technology permits the assessment of copy number alterations at a resolution of $1 \mathrm{~kb}$ and the detection of copy number-neutral loss of heterozygosity (CN-LOH) or uniparental disomy (UPD). Here we report the cytogenetic findings on a 7-year-old girl with growth hormone deficiency, short stature, learning difficulties and mild facial dysmorphism. Chromosome analysis and whole chromosome painting showed an interstitial duplication at 6q25.1q27. Chromosome microarray analysis (CMA) using Affymetrix 6.0 SNP platform has revealed this patient carries a 6.95 $\mathrm{Mb}$ interstitial triplication at 6q25.3q27 along with a 5.74-Mb segmental UPD located at the distal breakpoint of the triplication and extended to 6 qter. The triplicated region contains multiple reference genes listed in OMIM database including GTF2H5, IGF2R, LPA, PLG and PARK2. The terminal segmental UPD 6q27 also contains multiple genes but none is known to be imprinted. Parental study is ongoing. FISH assay using customized BAC probes mapping to the region of triplication to determine the orientation of the replicated genomic fragment is currently underway. Somatic recombination in the early stage of embryonic development is proposed to have medicated the above aberrations. This case has demonstrated the power of combining karyotype, FISH and state-of-art microarrays in detecting chromosome abnormalities which could not be achieved by any of these technologies alone. Such comprehensive cytogenetics/cytogenomics analysis will significantly improve the clinical outcome in cytogenetic laboratories and further help to illustrate the corresponding mechanisms for the aberrant chromosomal event.
50

Subjectivity in Chromosome Band Assessment

K. Geiersbach ${ }^{\mathrm{a}, \mathrm{b}}$, M. Sederberg ${ }^{\mathrm{a}}$, N. Uddin ${ }^{\mathrm{a}, \mathrm{b}}$, S. Shetty ${ }^{\mathrm{a}, \mathrm{b}}$

${ }^{a}$ ARUP Laboratories, Cytogenetics Laboratory, and bepartment of Pathology, University of Utah School of Medicine, Salt Lake

City, Utah, USA

Background: Chromatin condensation during prometaphase and metaphase results in a progressive decrease in the number of discernable chromosome bands. It is generally agreed upon that a higher band resolution permits the detection of more subtle chromosomal abnormalities. Assessment of band resolution is important in monitoring the quality of chromosome morphology and ensuring that laboratory procedures are optimized. Methods for assessing band resolution vary between cytogenetics laboratories in the U.S., and there is limited data on the accuracy and reproducibility of these methods. ISCN band level designation (300, $400,550,700,850)$ approximates the actual number of bands depicted in the idiograms $(299,387,555,747,857)$ [ISCN, 2009]. However, the actual number of countable bands in the chromosome images provided in ISCN 2009, designated as 500-900, is 321-652 (omitting acrocentric p arms) (pp 32-33). Methods: In order to standardize band resolution assessment in our laboratory, we compared 3 counting methods (Vancouver, Stallard, and Welborn) and 2 target band methods (Mayo and ACC-UK) on all karyotypes from each of 40 cases (20 amniotic fluid and 20 peripheral blood specimens). Results were compared to an exhaustive count of all chromosome bands on chromosomes 1-22 and X, omitting acrocentric $\mathrm{p}$ arms, and using the lower count on each homologue to determine the number of bands per haploid set. In order to determine reproducibility, individual karyotypes were evaluated by readers with different levels of experience. Results: Counting methods tended to be more stringent and yielded a lower band resolution in comparison to target band methods used on the same case. Target band methods showed better intra-observer and inter-observer reproducibility than counting methods. Conclusions: The presence or absence of chromosome bands that discriminate between a lower and higher band resolution is often uncertain because of subjectivity in band resolution assessment. Target band methods show greater reproducibility than counting methods and for this reason, target bands are preferable in assessing band level. Further studies are needed to demonstrate interlaboratory performance of target band systems, and standardization across U.S. laboratories is desirable. 


\section{1 \\ Small Genomic Abnormalities Observed by Array CGH Can Be Detected Using Oligonucleotide FISH Probes}

L.S. Rector ${ }^{a}$, N.A. Yamada ${ }^{\text {b }}$, M.C. Sederberg ${ }^{\text {a }}$, M.E. Aston ${ }^{\text {a }}$, R.A.Ach ${ }^{\mathrm{b}}$, P. Tsang ${ }^{\mathrm{b}}$, E. Carr ${ }^{\mathrm{b}}$, A. Scheffer-Wong ${ }^{\mathrm{b}}$, N. Sampas $^{\mathrm{b}}$, B. Peter ${ }^{\mathrm{b}}$, L. Bruhn $^{\mathrm{b}}$, A.R. Brothman ${ }^{\mathrm{a}, \mathrm{c}}$

${ }^{a}$ ARUP Laboratories, Salt Lake City, Utah, ${ }^{b}$ Agilent Laboratories, Agilent Technologies, Santa Clara, Calif., 'Departments of Pediatrics, Human Genetics and Pathology, University of Utah School of Medicine, Salt Lake City, Utah, USA

FISH (fluorescence in situ hybridization) is often used to confirm alterations in the genome detected by array comparative genomic hybridization (aCGH). Traditional FISH techniques use large probes $(150-300 \mathrm{~kb})$ generated from bacterial artificial chromosomes (BACs) to detect these alterations. Often, abnormalities detected by aCGH are either in genomic regions or of a genomic size which prohibit the use of this technique. In order to adapt FISH to resolutions afforded by aCGH, we developed methods to generate fluorescently labeled probes from oligonucleotide libraries, derived from massively parallel chemical synthesis. Sequences for oligonucleotide synthesis were selected in silico and include only the most informative elements of the loci, eliminating the need for suppressive hybridization reagents. Post-synthesis workflows involved PCR amplification, followed by the introduction of fluorescent labels by chemical modification. Here, we present the utility of these complex oligonucleotide library-derived FISH probes in further defining abnormalities detected by aCGH. The size of the loci range from $6 \mathrm{~kb}$ to $123 \mathrm{~kb}$, but after removing repetitive sequences and areas with high homology to non-target regions, probe coverage ranged between $3.6 \mathrm{~kb}$ to $40 \mathrm{~kb}$. We obtained robust fluorescent signals with a $94 \%$ success rate for 17 difficult genomic regions where traditional FISH failed. Small deletions and duplications were both detected. Oligonucleotide-based FISH is a powerful method for confirming abnormalities found by aCGH because it allows for higher resolution while sustaining sensitivity for visualization of smaller loci. Probes of this type can be generated for multiple research applications, utilizing virtually any DNA sequence.

\section{2 \\ Institutional Clinical Experience Using the Oligo-SNP Chromosomal Microarray \\ F. Quintero-Rivera ${ }^{\text {a }}$, W. Grody ${ }^{\text {a-c }}$, E. Baldwin ${ }^{\text {b }}$, S. Cederbaum ${ }^{\text {b, c }}$, B. Crandall ${ }^{\mathrm{b}}$, K. Dipple ${ }^{\mathrm{b}, \mathrm{c}}$, M. Fox ${ }^{\mathrm{b}}$, J.A. Martinez-Agosto ${ }^{\mathrm{b}, \mathrm{c}}$, J. Peredo ${ }^{\text {b }}$, E. Vilain ${ }^{\text {b-d }}$, X. Li $^{\text {a }}$, P.N. Rao ${ }^{\text {a }}$ \\ aDepartment of Pathology and Laboratory Medicine, ${ }^{b}$ Department of Pediatrics, 'Departments of Human Genetics, and ${ }^{\mathrm{d} D e p a r t m e n t}$ of Urology, David Geffen School of Medicine, University of California, Los Angeles, Calif., USA}

The screening of individuals with mental retardation and/or multiple congenital anomalies (MCA) as the predominant primary feature by Chromosomal Microarray Analysis (CMA) has resulted in the delineation of several new microdeletion/duplica- tion syndromes. CMA testing has aided to further delineate the subtle changes, particularly in those chromosomal regions that are technically difficult to identify. We report on our institutional experience using a Genome-Wide SNP oligonucleotide arraybased CMA platform (Affymetrix 6.0, Inc), which contains 1.8 million oligonucleotide DNA probes spaced at $\sim 696$ bp throughout the human genome, for copy number change (CNC) detection. The criteria for determination of a CNC consisted of 25 or more adjacent oligonucleotide probes covering a minimum of 50 $\mathrm{kb}$, or 50 or more probes covering $200 \mathrm{~kb}$, that showed at least a single copy number change.

A total of 226 cases have been analyzed since May 2008 $(5 / 1 / 08-2 / 1 / 10)$ with a yield of $18.1 \%$ abnormal results $(n=41 / 226)$. The majority of the results ( $\mathrm{n}=36 / 41,88 \%)$ overlapped with wellestablished disorders and recently described syndromes. The remaining $12 \%(n=5 / 41)$ are likely pathogenic but must be interpreted with caution because further studies are needed (parental CMA, PCR, etc). The different abnormalities detected and the minimum and maximum size range included: $7.3 \%$ loss of heterozygosity, ( $\mathrm{n}=3 / 41$; range $12 \mathrm{Mb}-150 \mathrm{Mb}$ ); $56.1 \%$ heterozygous deletions $(\mathrm{n}=23 / 41$; range $120 \mathrm{~kb}-13.4 \mathrm{Mb}) ; 29.3 \%$ duplications $(\mathrm{n}=12 / 41$; range $166 \mathrm{~kb}-45.2 \mathrm{Mb}) ; 2.4 \%$ triplication $(\mathrm{n}=1 / 41$; range $9.2 \mathrm{Mb}$ ); and $4.8 \%$ concurrent deletion and duplication $(\mathrm{n}=$ 2/41; size 32.8-45 Mb duplication, 0.3-1.2 Mb deletion). We also compared the results from these cases with other cytogenetic testing methods and found $51.2 \%$ cases had no previous karyotype (n $=21 / 41) ; 29.3 \%$ with previously studied normal karyotype $(\mathrm{n}=$ $12 / 41), 14.6 \%$ with an abnormal karyotype ( $n=6 / 41)$; and $7.3 \%$ with normal subtelomeric FISH $(n=3 / 41)$. Interestingly, of the abnormal cases with a deletion or duplication larger than $1 \mathrm{Mb}$, those that had a post-CMA high-resolution karyotype, the aberration was visible in three. Finally, the distribution of the different phenotypes associated with abnormal CMA results are more specifically defined [developmental delay (DD), DD plus 1 congenital anomaly/dysmorphic feature, 2 to $4 \mathrm{MCA},>5 \mathrm{MCA}$, autism, hypotonia (no DD), seizures].

\section{Poster Presentations Cancer Cytogenetics}

\section{3 \\ Whole Genomic Microarray Analysis of Pediatric Acute Leukemia Cases \\ L.S. Jenkins ${ }^{a}, X . L^{a}{ }^{a}$, V. Kiley ${ }^{b}$ \\ aThe Permanente Medical Group Regional Cytogenetics Laboratory, Northern Calif., ${ }^{\mathrm{b}}$ The Permanente Medical Group, Department of Pediatric Oncology, Roseville, Calif., USA}

Whole genomic microarray (aCGH) analysis, using a 105K oligonucleotide designed chip (Signature Genomics, LLC; Agilent Technologies), was performed on 13 pediatric acute leukemia cases; subtypes included precursor B-cell acute lymphoblastic

Preprint Cytogenet Genome Res 128/4/2010

Abstracts will be available online, free of charge 
leukemia (ALL), precursor T-cell ALL and acute myeloid leukemia (AML). The results of the aCGH analyses were compared with the standard results obtained by the chromosomal karyotyping and fluorescence in situ hybridization (FISH) methods by the Children's Oncology Group (COG) as a COG reference laboratory. The aCGH results were in close agreement with the findings of chromosomal gains and losses and unbalanced rearrangements observed by metaphase chromosomal analysis. In addition, aCGH revealed imbalances that were not readily identifiable by standard cytogenetic analyses. The presence of multiple gains of chromosomes was confirmed by aCGH in all previously identified hyperdiploid cases. aCGH detected an imbalance within 12 p13.2 at the site of the ETV6 gene in 3 cases which were either previously identified or confirmed by FISH. This ETV6 imbalance by aCGH was observed in 2 pre-B ALL cases and 1 AML case. Two AML cases with an $M L L$ rearrangement observed by FISH did not reveal any rearrangement in the $M L L$ region at $11 \mathrm{q} 23$ or the recipient location by aCGH. As expected, aCGH provided additional information, with the identification of submicroscopic imbalances that can be classified as clinically relevant, such as the loss of IKZF1 (Ikaros) at 7p12.2 and the loss of $P A X 5$ at $9 \mathrm{p} 13$. The most common imbalance observed in this collection of cases was the loss of CDKN2A at 9p21.3. This loss was observed in 4 of 9 pre-B ALL cases and in the 2 pre-T ALL cases. The 6 cases with the CDKN2A loss included 2 cases that demonstrated hyperdiploidy, 1 case with the ETV6-RUN rearrangement, 1 case with a complex karyotype, and 2 cases with normal results per standard cytogenetics. Additional submicroscopic imbalances were observed in other regions of the genome that may identify genes that contribute to tumorigenesis. These initial studies have demonstrated that whole genomic microarray analysis is a reliable method for the identification of both cytogenetically visible as well as submicroscopic imbalances in pediatric ALL and can be used in conjunction with existing methods to aid in the analysis of pediatric acute leukemia. The potential of this genome-wide approach to uncover and identify clinically relevant information demonstrates the need to collect this data along with the current standard methods in order to gain insights into the prognostic significance and ultimately into the pathogenesis and therapeutic approaches to this disease.

\section{4 \\ Cancer Predisposition Syndromes and Array Comparative Genomic Hybridization: When One Answer Does Not Tell the Whole Story}

R.D. Clark ${ }^{\text {a }}$, S. Ramanathan ${ }^{\text {a }}$, D. Michelson ${ }^{\text {b }}$

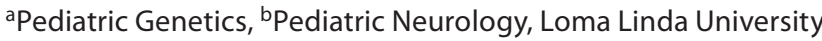
Children's Hospital, Loma Linda, Calif., USA

Cancer genetic syndromes and array comparative genomic hybridization $(\mathrm{aCGH})$ have posed unique genetic counseling challenges in the last decade. However, patients who have both a cancer predisposition syndrome and an aCGH anomaly are so rare that there is little to guide the clinician who counsels them. Our experience with 4 children with aCGH anomalies and cancer syndromes illustrate the risks and potential errors associated with counseling this group and the lack of aCGH reporting standards in this setting.

In each child, a deleted interval included a gene known to be associated with a cancer predisposition syndrome but in 2 patients, who had BAC aCGH, this was discovered only after a diligent and time-consuming pursuit by clinical genetic staff. These 4 patients were originally referred for developmental delay/mental retardation (DD/MR) and birth defects in three and learning disabilities in one. Their diagnoses were Peutz-Jeghers syndrome (PJS; STK11), neurofibromatosis 2 (NF2; NF2), Gorlin syndrome (GS; PTCH1) and Li-Fraumeni syndrome (LFS; p53). The diagnoses of NF2 and PJS preceded the aCGH results, which explained unusual findings: cleft palate and MR in the former and learning disabilities in the other. The GS and LFS diagnoses followed aCGH: the former had macrocephaly, post-axial polydactyly and $\mathrm{DD} / \mathrm{MR}$, the latter had DD/MR, thumb anomalies without a family history of cancer. These patients represent $\sim 10 \%$ of all children with array abnormalities in our clinic. Even as aCGH testing gains wide use, there are no agreed standards in the reporting and interpretation of abnormal/equivocal results. In 2 of our 4 patients, the test reports neither provided the genomic positions of the deleted clones nor mentioned significant genes deleted (PTCH1, p53). Clinical genetic providers and laboratory professionals need to work together to develop uniform guidelines for aCGH reports. Genes known to be associated with clinical findings must be listed in every report. These cases illustrate that single-gene disorders may be diagnosed incidentally with aCGH and further study is warranted when a child with a cancer genetic syndrome presents with unusual findings.

\section{5 \\ Chromosome Analysis Detects Two Cases of Donor Cell Disease following Allogeneic Bone Marrow Transplant \\ J. Malik ${ }^{\mathrm{a}}$, C. Miller ${ }^{\mathrm{b}}$, R. Blumer ${ }^{\mathrm{b}}$, E. Johnson ${ }^{\mathrm{b}}$, K. Thompson $^{\mathrm{b}}$, J. Northup b, J. Laffin ${ }^{\mathrm{a}, \mathrm{c}}$, G. Raca ${ }^{\mathrm{a}, \mathrm{b}}$ \\ aDepartment of Pathology and Laboratory Medicine, University of Wisconsin-Madison, bUW Cytogenetics Services, Wisconsin State Laboratory of Hygiene, 'Department of Pediatrics, University of Wisconsin-Madison, Madison, Wisc., USA}

Emergence of a hematological disorder in donor cells is an uncommon and under-reported complication after allogeneic hematopoietic stem cell transplant (HSCT). We report 2 cases where a disease of donor origin was detected by chromosome analysis during routine post-transplant follow-up.

The first patient is a 56-year-old woman diagnosed with $\mathrm{B}$ lymphoblastic leukemia in February of 2005. The bone marrow biopsy was markedly hypercellular (90\%) with diffuse involvement by B-cell lymphoblasts. She received a HSCT from her HLAmatched brother in July of 2005, and has been in morphological remission for 4 years. Interestingly, cytogenetic analysis of her most recent bone marrow biopsy revealed an abnormal male (donor) karyotype (7 out of 40 examined cells), with a deletion of the long arm of chromosome 20 from $20 \mathrm{q} 13.1$ to $20 \mathrm{q} 13.3$. The patient is currently well, with normal peripheral blood counts and nor- 
mocellular marrow demonstrating trilineage engraftment and maturation.

The second patient is a 50-year-old man diagnosed with chronic myelogenous leukemia in blast phase with associated fibrosis in September of 2007. He received a HSCT from his HLAmatched sister in April of 2009 and was in morphological and molecular remission until November, at which time cytogenetic analysis revealed an abnormal female (donor) karyotype (10 out of 40 examined cells) with a deletion of the long arm of chromosome 20 from $20 \mathrm{q} 11.2$ to $20 \mathrm{q} 13.1$. Subsequent bone marrow biopsy in January 2010 was markedly hypocellular with panhypoplasia and presence of the same cytogenetic abnormality.

In both described cases conventional cytogenetic analysis had a key role in early detection of the disease and confirmation of the donor cell origin. Although more than 40 cases of donor cell disease have been reported in the literature, the true prevalence of this complication and its etiology are still unknown. Various mechanisms have been proposed to explain malignant transformation of engrafted bone marrow cells, including aberrant homeostasis in the recipient bone marrow, sustained antigenic stimulation of lymphoid lineages, impaired immune surveillance and therapy-related mutagenesis. Future studies of donor cell malignancies may provide important insights into the mechanisms of induction, development, and progression of hematological diseases.

\section{6}

\section{Recurrent Interstitial Genomic Deletions of the Human PTEN Gene Are Facilitated by Flanking Microhomologies}

\author{
J.A Squire ${ }^{\mathrm{a}}$, M. Yoshimoto ${ }^{\mathrm{a}}$, A. Evans ${ }^{\mathrm{b}}$, K. Sircar ${ }^{\mathrm{c}}$, T. Bismar ${ }^{\mathrm{d}}$, \\ J. Williams ${ }^{\text {a }}$, P. Nuin ${ }^{\text {a }}$
}

${ }^{a} Q u e e n ' s$ University, Pathology and Molecular Medicine, Kingston, Ont., bUniversity Health Network, Pathology, Toronto, Ont., Canada; ' $U T$ MD Anderson Cancer Center, Pathology, Houston, Tex., USA; dUniversity of Calgary, Pathology \&

Laboratory Medicine and Oncology, Calgary, Alta., Canada

Genomic deletions of several hundred kb centering on the $P T E N$ gene are frequent in prostate cancer $(\mathrm{CaP})$. We have shown that hemizygous PTEN deletions in $\mathrm{CaP}$ are associated with earlier biochemical relapse, and that homozygous gene deletions are strongly linked to metastasis and androgen independent progression [Yoshimoto et al., 2007; Sircar et al., 2009]. These findings draw attention to the idea that pten protein dosage reduction is a major selective force of both onset and progression in $\mathrm{CaP}$. In this study we have mapped the location of CaP-specific PTEN deletions by applying 4 -color FISH methods for PTEN and several $\mathrm{kb}$ centromeric and telomeric regions to the gene on a cohort of 330 localized and hormone-refractory CaP tissue microarrays. PTEN genomic deletions were observed in $40 \%$ of $\mathrm{CaP}$ samples. FISH analyses showed that the most frequent loss at $10 \mathrm{q} 23$ was a recurrent interstitial, restricted to several hundred $\mathrm{kb}$ in size always including PTEN. The second most frequent class of deletion was more heterogeneous and involved loss of PTEN and the neighboring FAS locus. Variations of deletion size suggest that the $10 \mathrm{q} 23$ genomic region is highly unstable and is prone to $\mathrm{CaP}$-specific DNA rearrangements. Within the most frequently deleted region there is a fragile site (FRA10A) that may be involved in a subset of deletion events. Furthermore, bioinformatics analysis identified at least 3 clusters of non-redundant regions of microhomology surrounding the PTEN gene locus by intrachromosomal segmental duplications. We propose a model in which defects of homology-dependent DNA recombination and/or repair processes in prostatic somatic cells, lead to a propensity for prostate cancer precursors to undergo genomic PTEN deletion. The high incidence of deletion in these cells may be facilitated by error-prone recombinational repair events that utilize the segmental duplication and copy-number variation sequences. Collectively these data highlight the role of sequence microhomology clusters surrounding the PTEN gene locus on genomic stability in $\mathrm{CaP}$; and suggest that these regions may facilitate deletion events that lead to the decreased gene dosage of pten essential for $\mathrm{CaP}$ initiation and clinical progression.

\section{7 \\ Tetrasomy 13 as the Sole Chromosomal Abnormality in Acute Myeloid Leukemia}

C.M. Moore ${ }^{a}$, G.V.N. Velagaleti ${ }^{b}$, S. Vemulapallib

Departments of ${ }^{a}$ Cellular and Structural Biology and

bPathology, University of Texas Health Science Center at

San Antonio, Tex., USA

Trisomy 13 is a rare, but non-random, clonal abnormality associated with acute myeloid leukemia (AML) and other hematologic malignancies. It has been reported in more than 30 cases. However, isolated tetrasomy 13 has only been reported in 5 cases. These 5 patients presented with undifferentiated AML, advanced age, and rapid disease progression leading to death. Hence, isolated tetrasomy 13 is thought to represent an independent poor prognostic factor, especially in older individuals. We report an additional case of isolated tetrasomy 13 that further supports the association with a poor prognosis. An 85 -year-old male presented with fatigue and loss of appetite. He had a history of prostate cancer that was under control. Laboratory studies showed macrocytic anemia, thrombocytopenia, leukocytosis and numerous lymphoid-appearing blasts that expressed CD34, TdT, HLA-DR, partial CD117, CD13 and CD33. Based on these findings, he was diagnosed with AML with minimal differentiation (M0). Chromosomal analysis from the bone marrow aspirate showed $48, \mathrm{XY},+13,+13[20]$ as the only cytogenetic abnormality. Because of his age and the anticipated poor prognosis with or without chemotherapy, the patient opted for supportive care. He showed rapid disease progression and expired from pneumonia 3 weeks after the diagnosis. 


\section{8 \\ Variant Philadelphia Translocations in Chronic Myeloid Leukemia: Genesis and Prognosis}

M.M. Brahmbhatt, P.S. Patel, P.J. Trivedi, S.N. Shukla, P.M. Shah, S.R. Bakshi

Cell Biology Division, Medical Oncology, Department of Cancer Biology, The Gujarat Cancer \& Research Institute, Ahmedabad, India

The mechanisms for the formation of variant Philadelphia $(\mathrm{Ph})$ translocations that occur in $5-10 \%$ of patients with chronic myeloid leukemia (CML) are not fully characterized. Studies on the prognosis of these variant translocations have yielded conflicting results, especially regarding imatinib outcome and the status of deletions on the der(9). To shed light on these subjects, we sought to analyze all variant translocation cases identified at our institution.

Of 393 CML patients studied by conventional cytogenetics and fluorescence in situ hybridization (FISH), 2 showed masked $\mathrm{Ph}$. 16 patients (4\%) exhibited a variant $\mathrm{Ph}$. Besides chromosomes 9 and 22, chromosome 1 was the most frequently involved, at 3 times. Chromosomes 6, 11 and 13 were involved twice, whereas chromosomes 2, 3, 5, 7, 17 and 22 were involved once. Seventeen breakpoints were identified located preferentially in GC rich regions. Four patients showed novel rearrangements and 12 showed rare chromosomal abnormalities. Deletion on $\operatorname{der}(9)$ was observed in 4 (25\%) of the 16 cases, only 1 showed complete hematologic response, 2 showed partial and 1 showed no response, in all 4 there was no cytogenetic response observed. Higher prevalence was observed with 1-step mechanism in 10 (62\%), 2-step in 3 (18.75\%) and multiple-step in 3 (18.75\%). Out of three, 2 patients having 2-step mechanism showed complete hematologic response however expired during the course of treatment. In a patient with $\mathrm{t}(9 ; 22 ; 22)$, insertion of $A B L / B C R$ was observed on chromosome 22 , other than Ph. WCP FISH revealed insertion. BAC-FISH was performed to map the insertion breakpoint using the combination of RP11-339B21 (9q34, upstream to $A B L$ gene, spectrum green); along with 22q13.32 BACs CTA/bk299D3 and CTA/ bk799F1 (downstream to BCR gene, spectrum orange). BAC RP11-339B21 was also involved in the fusion, which consists mainly of 2 genes, URM1 and CERCAM. The result with CTA/ bk299D3 and RP11-339B21 showed 1 fusion and 1 orange signal on $\operatorname{der}(\underline{22})$ and a green signal for normal 9, whereas CTA/bk299D3 with RP11-339B21 showed 1 fusion and 1 orange signal on der(22), one split orange signal on $\operatorname{der}(9)$ and 1 green on normal 9.

Proper assessment of the prognostic significance of variant translocations requires better categorization based on their mechanisms of genesis and 9q34 deletion status as it may have clinical, prognostic and diagnostic implications.

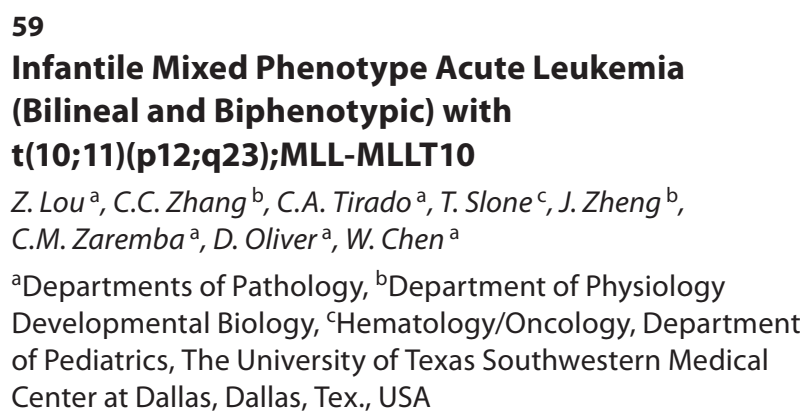

${ }^{\mathrm{a}}$ Departments of Pathology, ${ }^{\mathrm{b}}$ Department of Physiology Developmental Biology, 'Hematology/Oncology, Department of Pediatrics, The University of Texas Southwestern Medical Center at Dallas, Dallas, Tex., USA

We report a case of a 6-month-old boy with a mixed phenotype acute leukemia (MPAL), bilineal and biphenotypic immunophenotype (B-lymphoid lineage and combined B-lymphoid and monocytic lineage) with $\mathrm{t}(10 ; 11)(\mathrm{p} 12 ; \mathrm{q} 23) ;$ MLL-MLLT10. He was treated with acute myeloid leukemia protocol and in complete remission at 7-month follow-up. To the best of our knowledge, this is the first reported MLL-MLLT10 rearranged case presented as MPAL in an infant. From a practice standpoint, this case illustrates the importance of detection of $M L L$ rearrangement due to its prognostic implication and flow cytometry immunophenotyping in diagnosing MPAL and monitoring minimal residual disease.

\section{0 \\ Tumor Metastasis of Peromyscus leucopus Harderian Gland Tumors}

E.E. Mlynarski, C.J. Obergfell, M.J. O'Neill, R.J. O'Neill

University of Connecticut, Storrs, Conn., USA

The Peromyscus Genetic Stock Center has inbred lines of Peromyscus leucopus that are prone to spontaneous malignant Harderian gland tumors. A significant feature of the Peromyscus leucopus Harderian tumors is the rate and frequency of metastasis. These tumors are highly metastatic and immediately invade the lymphatic system, lungs and liver. Therefore, the Peromyscus leucopus tumor system provides an ideal, naturally occurring, in vivo model for investigating the mechanisms that drive metastatic potential.

We have employed cytogenetic and molecular techniques to identify the karyotypic and genetic signatures associated with metastasis of Peromyscus leucopus Harderian gland tumors. Preliminary work has identified a homogeneously staining region (hsr) on Chromosome 5 in Peromyscus leucopus primary Harderian gland tumors. This study will address the hypothesis that hsr 5 amplification contributes to the metastatic potential of Peromyscus leucopus Harderian gland tumors. 


\section{1 \\ Expanded FISH Assay Panel for Lymphoid Neoplasms Resulted Negative by Cytogenetics and Current FISH Panels but Positive by Hematopathology as a Reflex Test Rather than as a Part of Standard FISH Panels}

N.S. Mitter, S. Lanno, J. Blackson, M. Donskoy, R. Ehrenpreis

Dianon Systems (Laborp), Shelton, Conn., USA

Fluorescence in situ hybridization (FISH) panels for detecting lymphoid neoplasms in interphase nuclei currently used in most laboratories initially utilize the IGH (14q32.3) locus specific probe, and based on positive or negative results obtained, additional testing is done with either the MYC/IGH (8q24/14q32.3), CCND1/IGH (11q13/14q32.3), and IGH/BCL2 (14q32.3/18q22) locus specific probes, or the BCL6 (3q27), MYC (8q24), and MALT1 (18q21) locus specific probes, respectively. Although these probes detect a large number of lymphoma-related abnormalities, approximately $10 \%$ of cases are still negative by these FISH panels, but positive by hematopathology. In this pilot study including 27 patients, an expanded FISH panel was applied, utilizing the $A L K$ (2p23), MYB (6q23), and TCR $\alpha / \delta$ (14q11) probes. These probes were selected based on a high number of studies describing involvement of specific loci in gene rearrangements in lymphoid neoplasms. TCR $\alpha / \delta$ locus is frequently involved in gene rearrangements in T-cell lymphomas and leukemias. The MYB locus shows loss of heterozygosity in a high proportion of patients with peripheral T-cell and NK/T-cell lymphomas. The $A L K$ locus has also been well documented as involved in gene fusion with multiple partner loci. A known negative control for each probe and a known $M Y B$-positive control were used in a blind-coded set-up. This experimental expanded panel succeeded in detecting abnormalities in 4 of the $27(14.8 \%)$ patients included in this study. Three cases were positive for the loss of heterozygosity of the MYB locus, while 1 case was positive for involvement of the TCR $\alpha / \delta$ locus in a translocation. The $A L K$ probe did not detect any abnormalities. This pilot study clearly demonstrates the usefulness of incorporation of this expanded FISH panel. As opposed to incorporating these additional probes as a part of the standard panels, however, it seems to be more useful and cost-beneficial to have these as a reflex panel to the current standard panels for FISH studies in lymphoid neoplasms when the latter fail to detect abnormalities in cases with a positive hematopathology. We plan to continue investigation on additional patients meeting the criteria for inclusion in this study.

\section{2 \\ CIZ Gene Rearrangements in Pediatric CD10-Negative Acute Lymphoblastic Leukemia}

M. Shago a, c, G. Maire a , O. Abla b, d , J. Hitzler b, d, S. Weitzman b, d, M. Abdelhaleem ${ }^{\text {a, }}$ c

Departments of apaediatric Laboratory Medicine and

bPaediatrics, The Hospital for Sick Children, Departments of 'Laboratory Medicine and Pathobiology and dPediatrics, University of Toronto, Toronto, Ont., Canada

The CIZ (ZNF384) gene, located distal to the TEL (ETV6) gene at $12 \mathrm{p} 13.31$, is a putative zinc finger transcription factor which is recurrently rearranged in acute leukemia. To date, 23 patients with $C I Z$ gene rearrangement have been reported. Most of these patients are children or young adults with B-precursor acute lymphoblastic leukemia (ALL). Rearrangements of the CIZ gene result in attachment of various $5^{\prime}$ partner gene sequences to form $C I Z$ fusion genes. The CIZ gene has 3 known partners: TAF15 at $17 \mathrm{q} 12$ (16 cases), EWSR1 at 22q12 (4 cases), and E2A at $19 \mathrm{p} 13$ (3 cases). We present 7 new pediatric ALL patients with CIZ gene rearrangement. The patients, 5 females and 2 males, ranged in age at diagnosis from 2 to 15 years. All of our patients had lymphoblasts with a CD10-negative or CD10-low immunophenotype, similar to the antigenic profile seen in $M L L$ gene-rearranged ALLs. Follow up on the patients ranges from 10 to 40 months, and none of the patients have relapsed. These patients were diagnosed at our institution over the last 3.5 years. The $\mathrm{t}(12 ; 19)(\mathrm{p} 13 ; \mathrm{p} 13)$ and $\mathrm{t}(12 ; 22)(\mathrm{p} 13 ; \mathrm{q} 12)$ mediating the E2A-CIZ and EWSR1-CIZ translocations are difficult to identify by G-band analysis because the $C I Z, E 2 A$, and EWSR1 genes are near the distal ends of their respective chromosome arms. Identification of the rearrangements was facilitated using dual colour break-apart probes for the $E 2 A$, $C I Z$, and EWSR1 loci. Four of the patients had E2A-CIZ gene rearrangement and one had EWSR1-CIZ gene rearrangement. The remaining 2 patients had CIZ gene rearrangement involving novel regions on chromosomes 6 and 22, suggesting the presence of 2 additional $C I Z$ partner genes. During the time period of the study approximately 240 pediatric ALLs were analyzed, of which 40 were CD10-negative/low. Our data suggests that CIZ gene rearrangement may have an incidence of $\sim 3 \%$ in pediatric ALL, with an incidence of at least $18 \%$ in CD10-negative pediatric pre-B ALL. Since CIZ gene rearrangement may be associated with a more favorable prognosis than $M L L$ gene rearrangement, FISH analysis with probes to detect $C I Z$ gene rearrangement is recommended in patients with CD10-low/negative ALL.

Preprint Cytogenet Genome Res 128/4/2010

Abstracts will be available online, free of charge 


\section{3 \\ Detection of a Minimally Deleted Region of 6q21 in Chronic Lymphocytic Leukemia: Efficacy of BAC Array Data in Development of a Targeted 6q21 Fluorescence in situ Hybridization (FISH) Probe}

J.L. Murata-Collins, V. Bedell, D.B. Estrine, S.Y. Kim, Y.-H. Hsu

Department of Cytogenetics, City of Hope National Medical Center, Duarte, Calif., USA

Deletions of the long arm of chromosome 6 are recurrent findings in hematologic disorders, displaying variability in size and breakpoints. Suboptimal chromosome morphology and limited G-band resolution often confound accurate breakpoint determination for these deletions. If available, a FISH probe could potentially identify commonly deleted regions in hematologic disorders in general or $6 \mathrm{q}$ deletions characteristic of specific disease subgroups, such as chronic lymphocytic leukemia (CLL) versus acute lymphoblastic leukemia versus lymphoma versus myeloid disorders.

Historically, development of a FISH probe to detect a common region of deletion in the long arm of chromosome 6 would entail literature searches to find genes of interest, acquiring the corresponding BACs, PACs, or YACs, creating individual FISH probes and mapping each to confirm the chromosome location and specificity. Such a project previously had taken nearly 500 research hours, with no success. In contrast to the usual process, this project was completed in $\sim 70$ hours by using a RPCI-11 BAC DNA array. Ten DNA samples from a variety of hematologic malignancies containing a deletion of the long arm of chromosome 6 were analyzed, with a subset of CLL specimens showing a consistent region of deletion. Four BAC clones, including the 2 on the array identifying the region of deletion and 2 overlapping clones were obtained, generated into FISH probes, tested on positive and negative specimens, with a resulting probe spanning $790 \mathrm{~kb}$ mapped to a minimally deleted region of $6 \mathrm{q} 21$ in CLL. Of particular note, 1 of the 4 genes contained within the $6 \mathrm{q} 21$ probe is $\mathrm{FOXO} 3 \mathrm{~A}$, a transcription factor which plays a critical role in B-CLL cell resistance to apoptosis.

In summary, the speed and efficiency in developing the 6q21 FISH probe underscored the utility of using the BAC array as a primary screen to quickly define a critical region of interest in CLL. This approach holds the potential to define/refine other critical regions of gain or loss in other disease subgroups as well, enabling rapid generation of region-specific FISH probes with considerable savings of technical personnel hours over the traditional 'needle in the haystack' approach.

\section{4 \\ Chronic Lymphocytic Leukemia with $t(14 ; 19)(q 32 ; q 13)$ is Characterized by Atypical Morphologic, Immunophenotypic, and Genetic Features, and Stereotypy of the B-Cell Antigen Receptor}

L.V. Abruzzo a , C.D. Schweighofer ${ }^{a}$, R. Luthra ${ }^{a}$, R. Sargent ${ }^{\text {a }}$, R.P. Ketterling ${ }^{\text {C }}$, R.A. Knudson ${ }^{\text {C, L.L. Barron }}{ }^{\text {a }}$, M.J. Keating ${ }^{\text {b }}$, L.J. Medeiros ${ }^{\text {a }}$ Y.O. Huh ${ }^{\text {a }}$

aDepartments of Hematopathology and beukemia, The University of Texas M. D. Anderson Cancer Center, Houston, Tex., 'Division of Laboratory Genetics, Mayo Clinic, Rochester, Minn., USA

Introduction: The $\mathrm{t}(14 ; 19)(\mathrm{q} 32 ; \mathrm{q} 13)$ is a rare recurrent chromosomal translocation identified in various B-cell leukemias/ lymphomas, most commonly in chronic lymphocytic leukemia (CLL). This translocation usually juxtaposes BCL3 at 19q13 with the immunoglobulin heavy chain locus (IGH@) at 14q32. The clinicopathologic and genetic features of CLL with $t(14 ; 19)$ and IGH@/BLC3 rearrangement are poorly defined. In CLL, the repertoire of immunoglobulin heavy (IGHV) and light chain variable region gene segment use differs from that of healthy aging adults. Skewed gene segment use results in highly similar or 'stereotyped' sequences in the antigen binding regions of the monoclonal immunoglobulin molecule in subsets of CLL patients. This finding suggests that distinct (auto)antigens stimulate transformed B-cell clones that express specific antigen receptors encoded by a particular subset of immunoglobulin genes, and thereby promote the development of CLL. We describe the clinicopathologic and cytogenetic findings, and results of immunoglobulin gene sequence analysis in 14 CLL cases with $\mathrm{t}(14 ; 19)$ (q32;q13). Results: There were 10 men and 4 women, of median age 52.5 years (range 29-90). All patients presented with lymphocytosis; 10 had lymphadenopathy and 1 had splenomegaly. Lymphocytes in blood and bone marrow were predominantly small, but cytologically atypical. Immunophenotypic analysis by flow cytometry and immunohistochemical staining showed atypical immunophenotypes and expression of BCL3 in all cases. Conventional cytogenetic analysis demonstrated $t(14 ; 19)$ in all cases, associated with additional abnormalities in 10 cases, 7 with a complex karyotype. Trisomy 12 was identified in 9 cases. FISH using a dualcolor, dual-fusion probe demonstrated IGH@/BCL3 rearrangement in all cases. Sequence analysis of the $I G H V$ genes demonstrated that $13 / 14$ cases lacked somatic mutations, and 7 preferentially used IGHV segment 4-39. In 11 cases with adequate material, additional sequence analysis demonstrated homologous IGHV4-39 rearrangements in 7 cases and homologous kappa light chain VK1-39 rearrangements in 5 cases tested. Conclusions: Our results suggest that CLL with $\mathrm{t}(14 ; 19)(\mathrm{q} 32 ; \mathrm{q} 13)$ is a distinct subset with characteristic clinicopathologic and genetic features. Further, we are the first to demonstrate that a stereotyped B-cell antigen receptor is associated with a particular chromosomal translocation in CLL, which suggests that a specific antigen drives the clonal development and/or amplification of $\mathrm{t}(14 ; 19)$-positive CLL cells. 


\section{5}

Validation of FISH Studies on Chronic Lymphocytic Leukemia (CLL) by Members of the CLL Research Consortium.

S.A.Smoley ${ }^{\text {a }}$, D.L. Van Dyke a, N.E. Kay ${ }^{a}$, N.A. Heerema ${ }^{\text {b, }}$ M.L. dell' Aquila ', P. Dal Cin ${ }^{\text {d }}$, P. Koduru ${ }^{\text {e }}$, A. Aviram ${ }^{\mathrm{e}}$, L. Rassenti ${ }^{\mathrm{f}}$, J.C. Byrd ${ }^{\text {b }}$, K.R. Rai ${ }^{\text {g, J.R. Brown }}{ }^{\text {h }}$, A.W. Greaves ${ }^{c}$, T.J. Kipps ${ }^{\mathrm{c}}$ J. Eckel-Passow a , G.W. Dewald ${ }^{\mathrm{a}}$

aMayo Clinic, Rochester, Minn., bOhio State University, Columbus, Ohio, 'University of California San Diego, San Diego, Calif., 'Brigham \& Women's Hospital, Boston, Mass., ${ }^{\text {eNorth }}$

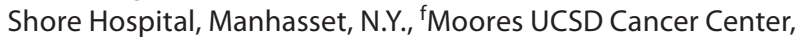
La Jolla, Calif., 'Long Island Jewish Med. Ctr., New Hyde Park, N.Y., ' Dana-Farber Cancer Inst., Boston, Mass., USA

Background: Five laboratories in the chronic lymphocytic leukemia (CLL) Research Consortium (CRC) investigated problems of pooling fluorescence in situ hybridization (FISH) results from clinical practice in their research. Methods: This investigation used fixed bone marrow and blood cells from prior conventional cytogenetic or FISH studies in 2 pilot studies, a 1-day workshop and proficiency test. Multiple FISH probe strategies were used to detect 6q-, 11q-, +12, 13q-, 17p- and t(14;?). Results: Ten specimens were studied by participants using their own probes (pilot study \#1). Of $300 \mathrm{FISH}$ interpretations, 218 (73\%) were true-negative, 68 (23\%) true-positive, 8 (3\%) false-negative and 6 (2\%) falsepositive. Each participant also studied 2 specimens using a shared FISH probe set for CLL (pilot study \#2). Of 80 FISH interpretations, no false-interpretations were identified. Workshop discussions produced agreement on scoring criteria, but a subsequent proficiency test for 2 specimens using participants own FISH probes produced 3 (4\%) of 67 false-positive interpretations. Conclusions: Inadequate normal cutoffs, inconsistent scoring criteria, and the use of different FISH probe sets contributed to interpretation errors. The normal cutoffs used among the CRC laboratories varied by as much as $5 \%$ and contributed to variation in the interpretation of specimens with a low level of nuclei with an abnormal signal pattern. For specimens that involve low percentages of abnormal nuclei, it would seem better to report false-negative interpretations for CLL FISH probes than it would be to report falsepositive interpretations. Thus, collaborative organizations that use pooled FISH results may need to impose their own higher normal cutoff values to eliminate false-positive interpretations.

\section{6}

\section{Chromosomal Aberrations in 48 Patients with Multiple Myeloma: Three-Year Study from a Single Institution in Singapore}

L.-C. Lau, P. Lim, G.-Y. Lee, L.-E. Loo, P.-S.F. Liaw, Y.-C.J. Lim,

L.-M. Teng, H.-S.K. See, T.-H. Lim, S.-T.A. Lim, D. Tan, S.-L. Tien

Department of Pathology, Cytogenetics Laboratory, Singapore General Hospital, Singapore

Aim: The incidence of multiple myeloma (MM) is lower in Asia than in western countries. Little is known if the cytogenetic abnormalities differ from those documented in the west. This study compared the chromosome abnormalities in our patient population with those derived from western populations. Material and Methods: Bone marrow samples from 71 patients with newly diagnosed MM between January 2007 and December 2009 were collected for cytogenetic study. The cultures were set up as direct cultures, 24-h cultures with overnight colcemid, and 72-h cultures with IL6. Results: Cytogenetic analyses revealed structurally and/or numerically abnormal clones in 48 patients (67.6\%). Hyperdiploidy (47 57 chromosomes) was found in 19 cases, hypodiploidy ( $<46$ chromosomes) in 17 patients, hypotriploidy (58 68 chromosomes) in 4 cases and hypotetraploidy (81 91 chromosomes) in another 4 patients. One case was hypertriploid (70 80 chromosomes) and the remaining 3 cases were pseudodiploid. Four primary breakpoints were recurrent: 1 p13 (11 cases; 23\%), 1q21 (9 cases; 18.8\%), 14q32 (7 cases; 14.5\%) and 6q13 (5 cases; $10.4 \%)$. Structural rearrangements of chromosome 1 were frequently observed, these include $\operatorname{del}(1 \mathrm{q})$, dup(1q), i(1q), and translocation with various partners. $\operatorname{Del}(1)(\mathrm{p} 13 \mathrm{p} 31)$ was the most common abnormality found ( 5 cases; $10.4 \%)$. The $t(11 ; 14)(\mathrm{q} 13 ; \mathrm{q} 32)$ rearrangement was seen in 2 cases while del(13)(q12q14) were reported in 3 cases. The most common numerical abnormalities were gains of chromosomes 15 (20 cases), 19 (17 cases), 9 (15 cases), 21 (12 cases), and gain of chromosomes 3, 5, 11 (11 cases each). Losses included chromosomes 13 (24 cases), 14 and X (13 cases each). Conclusion: The data showed that the chromosomal aberrations were often complex with hyperdiploidy as the most frequent finding. Among numerical changes, gains predominantly involved chromosomes 15, 19, 9, 21, 3, 5, 11 and the losses involved chromosomes 13, 14 and $\mathrm{X}$ (in descending order). There were also frequent structural rearrangements involving 1p13, 1q21, 14q32 and 6q13 (frequency in descending order). The study suggests that while chromosomal changes in our population showed some similarities with those seen in western populations, the $t(11 ; 14)$ and del13q14 is not as commonly seen in our population.

\section{7 \\ A Female Patient with FIP1L1-PGDFRA-Positive HES/CEL after Receiving R-CHOP Chemotherapy for Large Cell Lymphoma}

S. Kantarci ${ }^{\mathrm{a}, \mathrm{b}}$, M. Zhang ${ }^{\mathrm{a}}$, M. Gorman ${ }^{\mathrm{a}}$, G. Pihan ${ }^{\mathrm{a}, \mathrm{b}}$

aBeth Israel Deaconess Medical Center, Department of

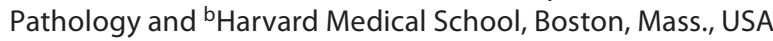

Hypereosinophilic syndrome (HES) and chronic eosinophilic leukemia (CEL) are rare hematologic disorders with persistent eosinophilia, tissue infiltration, and organ damage. Male to female ratio of HES/CEL is 9:1 with the mean age at onset 33 years. Recent identification of a $800-\mathrm{kb}$ interstitial deletion on chromosome $4 \mathrm{q} 12$ creating a FIP1L1-PDGFRA fusion gene with increased tyrosine kinase activity is the underlying cause of an important group of patients with HES/CEL and adds these disorder to the growing list of molecularly-defined chronic myeloproliferative disorders. Patients with FIP1L1-PGDFRA-positive disease have been reported to be very sensitive to the tyrosine kinase inhibitor imatinib mesylate.

A 60-year-old female patient was noted to have increased WBC and eosinophilia on routine follow up for treated diffuse 
large B cell lymphoma. She had initially been diagnosed in 2004 with an epidural mass at T3 to T5 level, treated with urgent laminectomy which revealed an extranodal non-Hodgkin B cell lymphoma. She received external beam radiation therapy to the thoracic spine. CT scan in 2005 showed increasing mesenteric adenopathy and thickening of the small bowel consistent with involvement by lymphoma. She received R-CHOP chemotherapy and had no evidence of disease at completion of consolidation therapy and autologous stem cell transplantation in 2006. Her bone marrow karyotype was normal: 46,XX [20]. Initial FISH studies excluded the abnormalities in $M L L, B C L 6, I G H, A B L$, and $B C R$ genes. In 2008 FISH for FIP1L1-PDGFRA fusion was performed with the LSI 4q12 Tri-Color Rearrangement Probe Set (Abbott Molecular) which uses 3 probes on chromosome band 4q12. The FIP1L1-PDGFRA fusion was observed in 97/100 nuclei obtained from bone marrow specimen.

She was started on Gleevec at the beginning of 2008 and is currently on $400 \mathrm{mg}$ daily. Her follow up FISH studies were negative for FIP1L1-PDGFRA fusion. Her eosinophil count has remained slightly elevated but stable. She has elevated CPK, which may be the effects from Gleevec or from the eosinophlia.

This case raises the tantalizing possibility that FIP1L1-PDGFRA fusions may rarely result from exposure to Topo II inhibitors, such as doxorubicin $\left(\right.$ Adriamycin $^{\circledR}$ ), which is part of R$\mathrm{CHOP}$ chemotherapy she received for her large cell lymphoma.

\section{8 \\ A Method for Achieving a 4-Hour Turnaround Time for FISH}

J.M. Cowan, K.A. Krajewski, S.C. Tillson, N.A. Kalgren, K.J. Connor

Cytogenetics Laboratory, Dept. Pediatrics, Tufts Medical Center, Boston, Mass., USA

In the laboratory we are frequently asked to confirm trisomy 21 and acute promyelocytic leukemia. We consider both to be rush indications for FISH and our turnaround time goal is 4 hours. Over the years we have abbreviated our sample preparation for FISH in order to maximize the hybridization time within our goal period. We have found that we can get excellent results with a fixed blood smear. This has allowed us to cut our preparation time to $15-25 \mathrm{~min}$, with hands-on time of about $5 \mathrm{~min}$. The method is manual and can be run at any time in our lab.

After a lesson in smear preparation from a colleague in Hematology, we use a Diff-safe ${ }^{\circledR}$ blood dispenser to get the blood from the tube to the slide. Once the smear has air-dried, we fix it in 50: 50 fix for a minimum of $10 \mathrm{~min}$ (ideally $20 \mathrm{~min}$ ). After a second air-drying, we use heat to co-denature the probe and slide $(51 / 2$ min in a Blue $\mathrm{M}$ oven at $90^{\circ} \mathrm{C}$ ). After 3-4 h we wash and score. For centromeric probes, shorter hybridization times are possible - $10 \mathrm{~min}$ is the minimum, but $2 \mathrm{~h}$ is sufficient for great results. The signals are as bright and large as those on conventional slides made from fixed pellets and run overnight.

This method allows us to provide answers in a very timely manner, and is greatly appreciated by our clinicians. It is also useful for those 5.30PM samples that need to be hybridized overnight.

\section{9 \\ Use of Microarray Technology (aCGH) in Chronic Lymphocytic Leukemia (CLL): Evaluation of Both Copy Number Changes and Relevant IGH Translations}

R. Schultz ${ }^{\text {, }}$ L.G.Shaffer ${ }^{\mathrm{a}}$, H. Greisman ${ }^{\mathrm{b}},{ }^{\text {, R. Tubbs }}{ }^{\mathrm{c}}$, K. Theil $^{\mathrm{c}}$, J. Cook ${ }^{c}$, T. Brown ${ }^{\text {d, B. Ballifa, M.L. Slovak }}{ }^{\text {a }}$

aSignature Genomic Laboratories, LLC, bUniversity of

Washington, ${ }^{\mathrm{C}} \mathrm{Cleveland}$ Clinic Foundation, and

${ }^{\mathrm{d}}$ CSI Laboratories, Spokane, Wash., USA

CLL is a highly variable disease with life expectancies ranging from months to decades. Cytogenetic findings play an integral role in defining the prognostic significance and treatment for individual patients. Deletion 13q14, trisomy 12, del(11q)/ATM, and $\operatorname{del}(17 \mathrm{p}) / \mathrm{TP} 53$ are among the most common abnormalities, and are associated with increasingly poorer prognosis in the order listed. Importantly, assessment of $I G H$ gene rearrangements is also emerging as a critical component of CLL diagnosis. We have developed an oligonucleotide microarray platform intended to provide (i) targeted coverage for clinically relevant regions, (ii) whole genome backbone coverage, and (iii) a means to detect balanced translocations. Evaluation by aCGH of 30 CLL cases with prior chromosome and/or FISH analysis was performed. A majority of those cytogenetic findings, representing each of the aforementioned diagnostic abnormalities, were identified and further delineated. The overall complexity of microarray findings correlated with the prognostic significance as samples bearing only 13q14 deletions exhibited few or no additional alterations and those with TP53 deletion exhibited complex results involving as many as 30 additional copy changes. Samples with trisomy 12 or ATM deletions displayed intermediate complexity. Clonality reflected by monoallelic versus biallelic deletion of 13q14 was apparent by aCGH and unbalanced rearrangements [e.g. 46,XY,add(8) (p11.2), add(11)(q13)] were recognized as large terminal losses for those chromosomes coupled with terminal gains of $7 p$ and $22 q$. Smaller aberrations detected were principally non-recurrent, but included significant findings such as deletion of LATS1, a negative regulator of TP53 shown to exhibit reduced expression in CLL. In addition to copy changes our studies have confirmed the utility of aCGH in detecting balanced translocations. Of relevance to CLL, IGH rearrangements with CCND1 $[\mathrm{t}(11 ; 14)]$ and BCL2 [t(14; 18)] were detected by the array in samples known to bear these translocations. In summary, assessment of CLL specimens by aCGH can identify aberrations commonly associated with this disease and through improved resolution, detect a significant number of unrecognized alterations that could yield future diagnostic and prognostic value. Moreover, we demonstrate that aCGH can be used to detect IGH translocations commonly assessed during the diagnosis of CLL. 